\title{
MEROMORPHIC DATA FOR MEAN CURVATURE ONE SURFACES IN HYPERBOLIC THREE-SPACE
}

\author{
RICARDO SA EARP AND ERIC TOUBIANA
}

(Received February 12, 2002, revised June 20, 2003)

\begin{abstract}
In this paper we construct meromorphic data and prove a representation theorem for mean curvature one conformal immersions into the hyperbolic three-space. We also give various examples.
\end{abstract}

Introduction. In this article we present a new approach to the theory of mean curvature one surfaces in the hyperbolic three-space. Our main result is the following: Every non-totally umbilic conformal immersion $X: U \rightarrow \boldsymbol{H}^{3}$ of a simply connected domain $U \subset \boldsymbol{C}$ into the half-space model of the hyperbolic three-space (denoted by $\boldsymbol{H}^{3}$ ), having mean curvature one with respect to the oriented euclidean Gauss map $E$ (Definition 1-1), gives rise to two meromorphic data $(h, T)$ defined on $U$ that describe completely $X$ (see Theorem 3-9).

Kenmotsu $[\mathrm{K}]$ showed that any $C^{2}$ solution on a simply connected domain $U$ of the equation

$$
E_{z \bar{z}}=2 \frac{\bar{E}}{1+E \bar{E}} E_{z} E_{\bar{z}}
$$

produces a conformal immersion $X: U^{*} \rightarrow \boldsymbol{R}^{3}$ of constant mean curvature, where $U^{*}=$ $U \backslash\left\{z ; E_{\bar{z}}=0\right\}$. He also proved a similar result for the case of prescribed mean curvature. As far as we know, however, no explicit (non-trivial) solutions of this equation are known.

In this work, we derive a similar equation, namely

$$
E_{z \bar{z}}=\frac{\bar{E}}{1+E \bar{E}} E_{z} E_{\bar{z}},
$$

for which every non-trivial solution gives rise to a mean curvature one conformal immersion $X: U^{*} \rightarrow \boldsymbol{H}^{3}$ into the hyperbolic three-space (Proposition 2-1 and Theorem 2-5). In contrast to the previous equation, we can give a complete description of the $C^{2}$-solutions of (*). Indeed, any solution of $(*)$ can be expressed in terms of meromorphic data $(h, T)$. Conversely, given any pair of non constant meromorphic functions $(h, T)$ with $h \neq 1 /(\alpha T+$ $\beta), \alpha, \beta \in C$, there is a natural way to describe explicitly a conformal parametrization of a piece of a surface with mean curvature one into the hyperbolic three-space, which involves

2000 Mathematics Subject Classification. Primary 53A35; Secondary 53A10.

Key words and phrases. Hyperbolic space, half-space model, mean curvature one conformal immersion, euclidean Gauss map, hyperbolic Gauss map.

The authors were partly suported by CNPq, FINEP and PRONEX of Brazil. The second author is grateful to the Mathematics Department of PUC-Rio, Brazil, for their hospitality during the elaboration of this paper. 
just one integration, $\int h^{2} T_{z} d z$. The branched points, if there are any, are isolated; in any case it is possible to handle the branched points and obtain many complete such surfaces. We emphasize that for mean curvature one immersion any geometric quantity can be expressed in terms of the euclidean Gauss map $E$ alone.

Besides, the authors shall present in Section 4, many conformally parametrized complete surfaces with mean curvature one, immersed into the hyperbolic three-space with certain geometric properties. We shall describe explicitely families of such surfaces invariant by a discrete subgroup (but not invariant by a one-parameter continuous subgroup) of each of the following group of rigid motions of $\boldsymbol{H}^{3}$ : parabolic, hyperbolic and elliptic.

This paper is organized as follows. We shall develop in Section 1 the theory of surfaces conformally immersed into $\boldsymbol{H}^{3}$ from our point of view. As a consequence, we shall derive the geometric quantities in terms of the euclidean Gauss map $E$ and the hyperbolic Gauss map $G$. We shall infer another proof of Bryant's result: $\tilde{H}=1$ if and only if $G$ is holomorphic, and the immersion is totally umbilic if and only if $G$ is anti-holomorphic. We observe that the latter astonishing result due to Bryant has thrown a light on the analytic nature of mean curvature one surfaces in $\boldsymbol{H}^{3}$. We note that Galvão and Góes [G-G] have also given an alternative proof of this theorem. We recall now that for minimal surfaces in euclidean space an analogous wellknown result holds. We shall explore the linking between minimal surfaces in euclidean space and mean curvature one surfaces in hyperbolic space over and over through our main results. This was explored before by Bryant [B], Umehara-Yamada [U-Y, 1] and the authors [SE-T, 1]. We begin Section 2 by establishing the globally defined equation for mean curvature one surfaces in $\boldsymbol{H}^{3}$, namely $(*)$, see Proposition 2-1. Then, we show that any solution of $(*)$ gives rise to a piece of a mean curvature one surface in the hyperbolic three-space, Theorem 2-5.

In Section 3 we shall develop our theory on mean curvature one surfaces, in order to prove our main results (Propositions 3-6, 3-7, 3-8). Finally, in Section 4, we shall give a family of complete examples.

Following Bryant idea's, Umehara and Yamada have introduced the notion of regular ends and provided several techniques, that have been very useful since then [U-Y, 1]. On this subject see also the following works: Umehara-Yamada [U-Y, 2], [U-Y, 3], RossmanUmehara-Yamada [R-U-Y, 1], [R-U-Y, 2], Lima-Rossman [L-R], Lima-Roitman [L-Roit], Rossman-Sato [R-S], the authors [SE-T, 1], and Collin-Hauswirth-Rosenberg [C-H-R].

The basic idea here is that, looking at the upper half-space model of hyperbolic space, one can see that hyperbolic geometry is very well integrated with Euclidean Geometry. This point of view has guided us and Barbosa to several papers (see [SE-T, 2], [SE-T, 3], [B-SE, 1] and [B-SE, 2]). We refer the reader to the authors'book as well [SE-T, 4].

We note that any conformal immersion in the half-space model of hyperbolic space can be expressed in terms of the euclidean Gauss map $E$ and the hyperbolic Gauss map $G$. In a forthcoming paper we make this more precise, see [SE-T, 5]. We note also that we have obtained a Weierstrass-Kenmotsu type theorem for prescribed mean curvature surfaces in hyperbolic space, see [SE-T, 6].

The authors are grateful to the referee for valuable observations. 
1. Surface theory in hyperbolic space. We begin by fixing the notation we shall use in this paper. We shall focus on the half-space model of the hyperbolic 3-space, which we shall denote by $\boldsymbol{H}^{3}$, namely

$$
\boldsymbol{H}^{3}=\left\{(u, v, w) \in \boldsymbol{R}^{3} ; w>0\right\}
$$

equipped with the hyperbolic metric

$$
\frac{d u^{2}+d v^{2}+d w^{2}}{w^{2}} .
$$

Throughout this paper, $U \subset \boldsymbol{C}$ will be a simply connected domain of the complex plane with coordinate $z=x+i y$, and $X: U \rightarrow \boldsymbol{H}^{3}$ will be a $C^{2}$ conformal immersion of $U$ into $\boldsymbol{H}^{3}$. We shall call $M=X(U)$ a surface in $\boldsymbol{H}^{3}$. For any vectors $\vec{u}$ and $\vec{v}$, the notation $\vec{u} \cdot \vec{v}$ (resp. $\langle\vec{u} ; \vec{v}\rangle$ ) stands for the standard euclidean (resp. hyperbolic) inner product of $\vec{u}$ and $\vec{v}$. Let $N$ be the euclidean Gauss map of $X$ such that $\left(X_{x}, X_{y}, N\right)(z)$ is a positively-oriented basis of $\boldsymbol{R}^{3}$ for each $z \in U$, where $X_{x}=\partial X / \partial x$ and $X_{y}=\partial X / \partial y$. That is,

$$
N=\frac{X_{x} \wedge X_{y}}{\left|X_{x} \wedge X_{y}\right|}
$$

where $|\cdot|$ stands for the euclidean norm and $\wedge$ for the euclidean vector product. We call $N=\left(N_{1}, N_{2}, N_{3}\right)$ the oriented euclidean Gauss map of $X$, or more briefly the euclidean Gauss map of $X$.

Definition 1-1. (1) Let $\Pi: S^{2} \rightarrow C \cup\{\infty\}$ be the standard stereographic projection. We set

so that

$$
E=\Pi \circ N=\frac{N_{1}+i N_{2}}{1-N_{3}}
$$

$$
N=\frac{(2 \operatorname{Re} E, 2 \operatorname{Im} E, E \bar{E}-1)}{E \bar{E}+1} .
$$

We call $E$ the oriented euclidean Gauss map of $X$.

(2) Let $p=X(z) \in M$ be a point on $M$. Let $\gamma^{+}$be the geodesic ray issuing from $p$, orthogonal to $M$ and oriented by the normal vector $N(z)$. Let $\omega \in \partial_{\infty} \boldsymbol{H}^{3}=C \cup\{\infty\}$ be the asymptotic boundary of $\gamma^{+}$. We then define a map $G: U \rightarrow C \cup\{\infty\}$ by setting $G(z)=\omega$. The map $G$ is the well-known hyperbolic Gauss map of $X$ (or $M$ ), $[B]$.

The following result states the relationship between these two Gauss maps $E$ and $G$, which will be fundamental throughout this work.

PROPOSITION 1-2. Using the notation above, we have

$$
u+i v=G-w E .
$$

Proof. Let $z \in U$ be any point. Observe that $G(z)=(u+i v)(z)$ implies that $N(z)=$ $(0,0,-1)$, and therefore $E(z)=0=(G-(u+i v))(z)$. Otherwise it suffices to express the equation of the geodesic ray. 
LEMma 1-3. Assume that $E(z) \neq \infty$ for any $z \in U$. Then we have

$$
w_{x}=2 \frac{\operatorname{Re}\left(\bar{E}\left(G_{x}-w E_{x}\right)\right)}{1+E \bar{E}}, \quad w_{y}=2 \frac{\operatorname{Re}\left(\bar{E}\left(G_{y}-w E_{y}\right)\right)}{1+E \bar{E}},
$$

that is,

$$
w_{\bar{z}}=\frac{\bar{E}}{1+E \bar{E}}\left(G_{\bar{z}}-w E_{\bar{z}}\right)
$$

where $w_{\bar{z}}=\partial w / \partial \bar{z}$.

Proof. As $N$ is the euclidean Gauss map of $X$, we have $X_{x} \cdot \Pi^{-1}(E)=X_{x} \cdot N=0$. Then, by Proposition 1-2, we obtain

$$
\begin{aligned}
(1+E \bar{E})\left(X_{x} \cdot N\right) & =\left((u+i v)_{x}, w_{x}\right) \cdot(2 E, E \bar{E}-1) \\
& =\left((G-w E)_{x}, w_{x}\right) \cdot(2 E, E \bar{E}-1) \\
& =2 \operatorname{Re}\left(\bar{E}\left(G_{x}-w E_{x}\right)\right)-w_{x}(E \bar{E}+1),
\end{aligned}
$$

which is the first equation of (1.1). The second equation of (1.1) is obtained in a similar way.

Lemma 1-4. Assume that $E(z) \neq \infty$ for any $z \in U$. Then we have

$$
G_{z}=w E_{z}
$$

and the (hyperbolic) metric $d s^{2}$ induced on $U$ by the immersion $X$ is given by

$$
d s^{2}=\frac{\left|G_{\bar{z}}-w E_{\bar{z}}\right|^{2}}{w^{2}}|d z|^{2} .
$$

Moreover, we have for every $z \in U$

$$
\bar{E}_{\bar{z}}(z) G_{\bar{z}}(z) \in \boldsymbol{R} .
$$

PROOF. As $X$ is a conformal immersion, we have

$$
X_{x} \cdot X_{x}=X_{y} \cdot X_{y} \text { and } X_{x} \cdot X_{y}=0 .
$$

On the other hand, using Proposition 1-2 and Lemma 1-3, we have

$$
\begin{gathered}
X_{x} \cdot X_{x}=\left|G_{x}-w E_{x}\right|^{2} \text { and } X_{y} \cdot X_{y}=\left|G_{y}-w E_{y}\right|^{2}, \\
X_{x} \cdot X_{y}=\operatorname{Re}\left(\left(G_{x}-w E_{x}\right)\left(\bar{G}_{y}-w \bar{E}_{y}\right)\right) .
\end{gathered}
$$

From (1.6)-(1.8) we deduce that complex numbers $\left(G_{x}-w E_{x}\right)$ and $\left(G_{y}-w E_{y}\right)$ have the same modulus and their arguments differ from $\pm \pi / 2$. Thus, we get $\left(G_{y}-w E_{y}\right)=$ $\pm i\left(G_{x}-w E_{x}\right)$. Now recall that

$$
N=\frac{X_{x} \wedge X_{y}}{\left|X_{x} \wedge X_{y}\right|}=\frac{(2 E, E \bar{E}-1)}{E \bar{E}+1} .
$$

Therefore, by Lemma 1-3, we get $\left(G_{y}-w E_{y}\right)=-i\left(G_{x}-w E_{x}\right)$. Hence we have $G_{z}-w E_{z}=$ 0 . 
Now, as $X$ is a conformal immersion, the metric $d s^{2}$ induced on $U$ by $X$ is given by

$$
d s^{2}=\frac{X_{x} \cdot X_{x}}{w^{2}}\left(d x^{2}+d y^{2}\right)=\frac{\left|G_{x}-w E_{x}\right|^{2}}{w^{2}} \cdot|d z|^{2} .
$$

On account of $G_{\bar{z}}-w E_{\bar{z}}=G_{\bar{z}}-w E_{\bar{z}}+G_{z}-w E_{z}=G_{x}-w E_{x}$, we have (1.4). lemma.

Finally, as $w_{x y}=w_{y x}$, by Lemma 1-3, we get (1.5), which completes the proof of the

Definition 1-5. (1) We call $\tilde{N}$ the unit normal vector field on $M$ with respect to the hyperbolic norm, having the same orientation as $N$, if $\tilde{N}=w N$.

(2) Denote by $D$ (resp. $\nabla$ ) the Riemannian connection of $\boldsymbol{R}^{3}$ (resp. $\boldsymbol{H}^{3}$ ). Also, denote by $\Pi$ (resp. $\tilde{\Pi}$ ) the euclidean (resp. hyperbolic) second fundamental form of the immersion $X$ with respect to $N$ (resp. $\tilde{N}$ ), that is,

$$
\Pi=b_{x x} d x^{2}+2 b_{x y} d x d y+b_{y y} d y^{2} \text { and } \tilde{\Pi}=\tilde{b}_{x x} d x^{2}+2 \tilde{b}_{x y} d x d y+\tilde{b}_{y y} d y^{2} .
$$

For example, $b_{x x}=-X_{x} \cdot N_{x}$ and $\tilde{b}_{x x}=-\left\langle X_{x}, \nabla_{X_{x}} \tilde{N}\right\rangle$. Recall also that for any vector fields $A, B$ and $C$ we have

$$
\left\langle C, \nabla_{B} A\right\rangle=\frac{1}{w^{2}} C \cdot D_{B} A+\frac{1}{w^{3}}(-A[w] B \cdot C-B[w] C \cdot A+C[w] A \cdot B),
$$

where $A[w]$ stands for the (euclidean) derivative of $w$ with respect to $A$, see [N] or [B-SE, 2].

(3) We denote by $H$ (resp. $\tilde{H}$ ) the euclidean (resp. hyperbolic) mean curvature of $X$ with respect to $N$ (resp. $\tilde{N}$ ). That is,

$$
H=\frac{b_{x x}+b_{y y}}{2 X_{x} \cdot X_{x}}, \quad \tilde{H}=\frac{\tilde{b}_{x x}+\tilde{b}_{y y}}{2 X_{x} \cdot X_{x}} .
$$

(4) We denote by $\Phi$ (resp. $\tilde{\Phi}$ ) the Hopf function of $X$ in $\boldsymbol{R}^{3}$ (resp. $\boldsymbol{H}^{3}$ ), namely

$$
\Phi=\frac{b_{x x}-b_{y y}}{2}-i b_{x y}, \quad \tilde{\Phi}=\frac{\tilde{b}_{x x}-\tilde{b}_{y y}}{2}-i \tilde{b}_{x y} .
$$

LEMмa 1-6. Assume that $E(z) \neq \infty$ for any $z \in U$. Then we have

$$
\begin{gathered}
H=\frac{-2 E_{\bar{z}}}{(1+E \bar{E})\left(G_{\bar{z}}-w E_{\bar{z}}\right)}, \\
2 \frac{G_{\bar{z}}}{1+E \bar{E}}=(1-\tilde{H})\left(G_{\bar{z}}-w E_{\bar{z}}\right), \\
H G_{\bar{z}}=(\tilde{H}-1) E_{\bar{z}}, \\
\Phi=-2 E_{z} \frac{\overline{G_{\bar{z}}-w E_{\bar{z}}}}{1+E \bar{E}}, \\
\tilde{\Phi}=-2 E_{z} \frac{\overline{G_{\bar{z}}-w E_{\bar{z}}}}{w(1+E \bar{E})}=\frac{1}{w} \Phi .
\end{gathered}
$$


PROOF. By Lemma 1-3, we easily get

$$
-b_{x x}=X_{x} \cdot N_{x}=\frac{2}{1+E \bar{E}} \operatorname{Re}\left(\bar{E}_{x}\left(G_{x}-w E_{x}\right)\right) .
$$

Now, using the relation (1.9), we thus get

$$
\begin{aligned}
\tilde{b}_{x x} & =-\left\langle X_{x}, \nabla_{X_{x}} \tilde{N}\right\rangle=\frac{1}{w^{2}}\left[w b_{x x}+N_{3} X_{x} \cdot X_{x}\right] \\
& =\frac{1}{w^{2}}\left[\frac{-2 w}{1+E \bar{E}} \operatorname{Re}\left(\bar{E}_{x}\left(G_{x}-w E_{x}\right)\right)+\frac{E \bar{E}-1}{E \bar{E}+1}\left|G_{x}-w E_{x}\right|^{2}\right] .
\end{aligned}
$$

The other relations involving $b_{x y}, b_{y y}, \tilde{b}_{x y}$ and $\tilde{b}_{y y}$ can be shown in a similar way.

Now relations (1.10)-(1.14) are easily inferred by using the definition of $H, \tilde{H}, \Phi$ and $\tilde{\Phi}$ and (1.5). This concludes the proof of the lemma.

REMARK 1-7. It is well-known that every positive isometry (that is, an isometry preserving the orientation) $J: \boldsymbol{H}^{3} \rightarrow \boldsymbol{H}^{3}$ extends continuously to the asymptotic boundary $\partial_{\infty} \boldsymbol{H}^{3}$ of $\boldsymbol{H}^{3}$. Moreover, the restriction of $J$ to $\partial_{\infty} \boldsymbol{H}^{3}$, denoted by $J_{\infty}$, is a Möbius function. Conversely, each Möbius function on $\partial_{\infty} \boldsymbol{H}^{3}$ is the restriction of a unique positive isometry of $\boldsymbol{H}^{3}$. Let us call $\zeta=u+i v$ the coordinate on $\partial_{\infty} \boldsymbol{H}^{3}=\boldsymbol{C} \cup\{\infty\}$. Note that every Möbius function $f$ on $C \cup\{\infty\}$ has a form of either

$$
f(\zeta)=\lambda e^{i \theta} \zeta+\beta, \quad \text { or } \quad f(\zeta)=\alpha+\frac{\lambda e^{i \theta}}{\zeta+\beta},
$$

where $\alpha, \beta \in C$ and $\lambda, \theta \in R, \lambda>0$.

LeMma 1-8. Let $J: \boldsymbol{H}^{3} \rightarrow \boldsymbol{H}^{3}$ be a positive isometry and $J_{\infty}$ the restriction of $J$ to $\partial_{\infty} \boldsymbol{H}^{3}$. Let $\hat{E}$ be the euclidean Gauss map of the immersion $\hat{X}=J \circ X: U \rightarrow \boldsymbol{H}^{3}$. Then we have

$$
\begin{gathered}
\hat{E}=e^{i \theta} \cdot E \quad \text { if } J_{\infty}(\zeta)=\lambda e^{i \theta} \cdot \zeta+\beta \\
\hat{E}=\frac{e^{i \theta}}{G+\beta}\left(G_{z} \frac{1+E \bar{E}}{E_{z}}-E \overline{(G+\beta)}\right) \quad \text { if } J_{\infty}(\zeta)=\alpha+\frac{\lambda e^{i \theta}}{\zeta+\beta} .
\end{gathered}
$$

Proof. For any complex number $\beta$ and any real numbers $\lambda, \theta, \lambda>0$, we denote by $H_{\lambda}$ the homothety on $\boldsymbol{H}^{3}$ with respect to 0 and ratio $\lambda$, by $T_{\beta}$ the horizontal $(\operatorname{Re} \beta, \operatorname{Im} \beta, 0)$ euclidean translation on $\boldsymbol{H}^{3}$, and by $R_{\theta}$ the euclidean rotation on $\boldsymbol{H}^{3}$ with respect to the $w$-axis with argument $\theta$. Observe that $H_{\lambda}, T_{\beta}$ and $R_{\theta}$ are positive isometries of $\boldsymbol{H}^{3}$.

Suppose that $J_{\infty}(\zeta)=\alpha+\lambda e^{i \theta} /(\zeta+\beta)$. Let $I: \boldsymbol{H}^{3} \rightarrow \boldsymbol{H}^{3}$ be the positive isometry of $\boldsymbol{H}^{3}$ such that $I_{\infty}(\zeta)=1 / \zeta$. Then we have $J=T_{\alpha} \circ H_{\lambda} \circ R_{\theta} \circ I \circ T_{\beta}$. Set $J \circ X=\hat{X}=(\hat{u}, \hat{v}, \hat{w})$ and consider the hyperbolic Gauss map $\hat{G}$ of $\hat{X}$. Thus $\hat{G}=J_{\infty}(G)$. Observe also that

$$
I(\zeta, w)=\frac{(\bar{\zeta}, w)}{|\zeta|^{2}+w^{2}} .
$$


Then we obtain

$$
J(\zeta, w)=(\alpha, 0)+\lambda \frac{\left(e^{i \theta}(\overline{\zeta+\beta}), w\right)}{|\zeta+\beta|^{2}+w^{2}}, \quad \hat{G}=\alpha+\frac{\lambda e^{i \theta}}{G+\beta} .
$$

Now, the proof is a straightforward verification by using Proposition 1-2, Lemma 1-4 and the relation $\hat{E}=(\hat{G}-(\hat{u}+i \hat{v})) / \hat{w}$.

The following result was first proved by Bryant [B, Proposition 1]. See also Galvão and Góes [G-G] for an alternative proof.

Corollary 1-9. (1) Let $p=X\left(z_{0}\right) \in M$ such that $E\left(z_{0}\right) \neq \infty$. Then $p$ is an umbilic point of $M$ if and only if $G_{z}\left(z_{0}\right)=0$. Therefore, $M$ is totally umbilic if and only if $G_{z} \equiv 0$, that is, $G$ is anti-meromorphic.

(2) Let $p=X\left(z_{0}\right) \in M$ such that $E\left(z_{0}\right) \neq \infty$. Then $\tilde{H}\left(z_{0}\right)=1$ if and only if $G_{\bar{z}}\left(z_{0}\right)=0$. Therefore, $\tilde{H} \equiv 1$ if and only if $G$ is meromorphic.

Consequently, the umbilic points of any non-totally umbilic mean curvature one surface in $\boldsymbol{H}^{3}$ are isolated.

PROOF. From (1.3) and the fact that $w>0$ it follows that for any $z_{0} \in U$ such that $E\left(z_{0}\right) \neq \infty$, we have $G_{z}\left(z_{0}\right)=0$ if and only if $E_{z}\left(z_{0}\right)=0$, which occurs if and only if $p=X\left(z_{0}\right)$ is an umbilic point.

Now, let $z_{0} \in U$ be a point such that $E\left(z_{0}\right)=\infty$. Let $J: \boldsymbol{H}^{3} \rightarrow \boldsymbol{H}^{3}$ be a positive isometry of $\boldsymbol{H}^{3}$ such that $\hat{E}\left(z_{0}\right) \neq \infty$, where $\hat{E}$ is the oriented euclidean Gauss map of the immersion $\hat{X}=J \circ X$. Let $J_{\infty}$ be the restriction of $J$ to the asymptotic boundary $\partial_{\infty} \boldsymbol{H}^{3}$. For the hyperbolic Gauss map $\hat{G}$ of $\hat{X}$, we have $\hat{G}=J_{\infty} \circ G$. We see that $z_{0}$ is an umbilic point of $X$ if and only if $z_{0}$ is an umbilic point of $\hat{X}$, which occurs if and only if $\hat{G}_{z}\left(z_{0}\right)=0$ so that $G$ is anti-meromorphic at $z_{0}$. From this we easily deduce the last statement in assertion (1).

In the same way, since the metric (1.4) does not degenerate, we have $G_{\bar{z}}-w E_{\bar{z}} \neq 0$ on $U$. Then, we deduce from (1.11) that $G$ is holomorphic at $z_{0}$ if and only if $\tilde{H}\left(z_{0}\right)=1$ on $U$. In case where there exist some points $z_{0} \in U$ such that $E\left(z_{0}\right)=\infty$, we proceed as before to conclude that $\tilde{H} \equiv 1$ if and only if $G$ is a meromorphic map. This completes the proof of the corollary.

We deduce from Lemma 1-6 the following equation for constant mean curvature.

COROLlary 1-10. Assume that $X: U \rightarrow \boldsymbol{H}^{3}$ is a $C^{3}$ conformal immersion such that $E(z) \neq \infty$ for any $z \in U$. Then $\tilde{H}$ is constant if and only if

$$
(1+E \bar{E}) E_{z \bar{z}}-\bar{E} E_{z} E_{\bar{z}}-\bar{E} E_{z} \frac{G_{\bar{z}}}{w}=0 .
$$

Proof. Since $G_{\bar{z}}-w E_{\bar{z}} \neq 0,(1.11)$ leads to

$$
\tilde{H}-1=-2 \frac{G_{\bar{z}}}{(1+E \bar{E})\left(G_{\bar{z}}-w E_{\bar{z}}\right)} .
$$


Here, since $\tilde{H}$ is real, $\tilde{H}$ is constant if and only if $\tilde{H}_{z}=0$, which is equivalent to

$$
\begin{aligned}
& w(1+E \bar{E}) E_{\bar{z} z}\left(G_{\bar{z}}-w E_{\bar{z}}\right)-w \bar{E} E_{\bar{z}} E_{\bar{z}}\left(G_{\bar{z}}-w E_{\bar{z}}\right) \\
& \quad-G_{\bar{z}}\left(E_{\bar{z}} \bar{E}+E(\bar{E})_{\bar{z}}\right)\left(G_{\bar{z}}-w E_{\bar{z}}\right)+E E_{\bar{z}} G_{\bar{z}} \overline{\left(G_{\bar{z}}-w E_{\bar{z}}\right)}=0 .
\end{aligned}
$$

Then by (1.5) we have the conclusion.

2. Mean curvature one surfaces in $H^{3}$ via the euclidean Gauss map. Throughout this section we shall use the same notation as in Section 1. From now on we assume that $X: U \rightarrow \boldsymbol{H}^{3}$ is a $C^{3}$ conformal immersion, where $U \subset \boldsymbol{C}$ is a simply connected domain.

By Corollaries 1-9 and 1-10, we have the following

Proposition 2-1. Assume that $\tilde{H} \equiv 1$ and $E(z) \neq \infty$ for any $z \in U$. Then

$$
E_{z \bar{z}}=\frac{\bar{E}}{1+E \bar{E}} E_{z} E_{\bar{z}} \text {. }
$$

REMARK 2-2. (1) Assume that $X$ is not a totally umbilic immersion and $E(z) \neq \infty$ for any $z \in U$. Then we also have the following necessary and sufficient condition for $\tilde{H} \equiv 1$ :

$$
w_{\bar{z}}=-w \frac{\bar{E} E_{\bar{z}}}{1+E \bar{E}} .
$$

Indeed, if $\tilde{H} \equiv 1$, then (2.1) holds because of Lemma 1-3 and Corollary 1-9.

Reciprocally, suppose that (2.1) holds. From Lemma 1-3 we infer that $\bar{E} G_{\bar{z}} \equiv 0$. Set $U_{1}=\{z \in U ; E(z) \neq 0\}$ and $U_{2}=\left\{z \in U ; G_{\bar{z}}(z) \neq 0\right\}$. Thus $U_{1}$ and $U_{2}$ are open subsets of $U$ such that $U_{1} \cup U_{2}=U$. Hence we have the following alternative: $U_{1}=U$ or $U_{2}=U$ or $U_{1} \cap U_{2} \neq \emptyset$. Note that, if $U_{1} \neq \emptyset$, then we have $G_{\bar{z}}=0$ on $U_{1}$ so that $\tilde{H} \equiv 1$ on $U_{1}$. Furthermore, if $U_{2} \neq \emptyset$, then $E=0$ on $U_{2}$, that is, $X\left(U_{2}\right)$ is a peace of a horizontal euclidean plane in $\boldsymbol{H}^{3}$ with downward normal, which implies that $\tilde{H} \equiv-1$ on $U_{2}$. These observations force $U_{1} \cap U_{2}=\emptyset$. Note also that $U_{1}=\emptyset$ implies that $E \equiv 0$ on $U$, and hence $X$ is a totally umbilic immersion, which is a contradiction. Consequently, $U_{2}=\emptyset$, that is, $G_{\bar{z}}=0$ on $U$. We therefore conclude that $\tilde{H} \equiv 1$ on $U$.

(2) Observe that Proposition 2-1 shows that Equation $(*)$ is a necessary condition for $\tilde{H} \equiv 1$ but is not a sufficient condition.

Indeed, assume that $X$ is a mean curvature one conformal immersion such that $E \neq \infty$ on $U$. Then its euclidean Gauss map $E$ satisfies Equation $(*)$. Let $\lambda>0$ and consider the new conformal immersion $X_{1}=X+(0,0, \lambda)$. Clearly, $X_{1}$ and $X$ have the same euclidean Gauss map. Therefore the euclidean Gauss map of $X_{1}$ satisfies Equation (*). Note that vertical translations are not isometries of $\boldsymbol{H}^{3}$. It is easy to deduce that $X_{1}$ is not a mean curvature one immersion into $\boldsymbol{H}^{3}$. For instance, any positive vertical translation of any horosphere whose asymptotc boundary is a finite point in $\partial_{\infty} \boldsymbol{H}^{3}$ (that is, any euclidean sphere in $\boldsymbol{H}^{3}$ tangent to $\{w=0\})$ is a compact sphere in $\boldsymbol{H}^{3}$ whose mean curvature with respect to the inward unit normal vector field is strictly bigger than 1 . 
Similarly, suppose that $X$ is a mean curvature one conformal immersion with $E \neq \infty$ on $U$, such that the coordinate $w$ is bounded from above. Then there exists a $\gamma>0$ such that $w(z)<\gamma$ for every $z \in U$. Therefore $X_{2}:=-X+(0,0, \lambda): U \rightarrow \boldsymbol{H}^{3}$ is a conformal immersion for any $\lambda \geq \gamma$. The oriented euclidean Gauss map of $X_{2}$ is $E$, which satisfies Equation $(*)$ although the mean curvature of $X_{2}$ is not one.

As a matter of fact, let $X: U \rightarrow \boldsymbol{H}^{3}$ be a conformal $C^{3}$ immersion such that $E(z) \neq \infty$ for any $z \in U, E$ satisfies Equation $(*)$ and $\tilde{H} \not \equiv 1$. Then we prove in a forthcoming paper, see [SE-T, 5], that $X$ must have one of the two forms mentioned above unless $E_{\bar{z}} \equiv 0$, that is, $X$ is a minimal immersion into $\boldsymbol{R}^{3}$ in euclidean meaning.

(3) Remark that Equation $(*)$ does not depend on the choice of a complex coordinate.

Lemma 2-3. Let $E: U \rightarrow C$ be a $C^{2}$ function satisfying (*). Then the set $\{z \in$ $\left.U ; E_{z}(z)=0\right\}$ is discrete unless $E$ is anti-holomorphic on $U$. Similarly, $\left\{z \in U ; E_{\bar{z}}(z)=0\right\}$ is a discrete set unless $E$ is holomorphic on $U$.

Proof. Let us first show that $\left\{z \in U ; E_{z}(z)=0\right\}$ is a discrete subset of $U$. For this, we are going to show the existence of a positive real function $\varphi: U \rightarrow \boldsymbol{R}^{+}$such that $\varphi E_{z}$ is a holomorphic function.

For any real function $\varphi$ on $U, \varphi E_{z}$ is holomorphic if and only if $\left(\varphi E_{z}\right)_{\bar{z}}=0$, that is, if and only if

$$
\left(\varphi_{\bar{z}}+\varphi \frac{\bar{E} E_{\bar{z}}}{1+E \bar{E}}\right) E_{z}=0,
$$

since $E$ satisfies Equation (*). Therefore,

$$
\frac{\varphi_{\bar{z}}}{\varphi}=-\frac{\bar{E} E_{\bar{z}}}{1+E \bar{E}}
$$

is a sufficient condition for $\varphi E_{z}$ being holomorphic. Set $f=(\log \varphi)$ and consider the equation

$$
f_{\bar{z}}=-\frac{\bar{E} E_{\bar{z}}}{1+E \bar{E}} .
$$

The integrability condition of (2.3) is given by

$$
\operatorname{Im}\left(\left(\frac{\bar{E} E_{\bar{z}}}{1+E \bar{E}}\right)\right)=0
$$

which is satisfied, since $E$ satisfies Equation (*). Since $U$ is a simply connected domain, we deduce that there exists a real function $f$ on $U$ which is a solution of (2.3), so that

$$
f=-2 \operatorname{Re} \int\left(\frac{\bar{E} E_{\bar{z}}}{1+E \bar{E}}\right) d \bar{z} .
$$

Thus the fonction $\varphi=e^{f}$ is a positive real function satisfying (2.2). Therefore $\varphi E_{z}$ is a holomorphic function as desired.

The second part is shown in the same way. 
LEMMA 2-4. Let $X$ be $a C^{3}$ non-totally umbilic conformal immersion such that $E(z) \neq$ $\infty$ for any $z \in U$. Assume that its euclidean Gauss map E satisfies Equation (*) and that $G_{z}=w E_{z}$ is a holomorphic map. Then we have $\tilde{H} \equiv 1$.

PROOF. Indeed, as $w E_{z}$ is a holomorphic map, $w_{\bar{z}} E_{z}+w E_{z \bar{z}}=0$. Since $E$ satisfies Equation (*), we then get

$$
w_{\bar{z}} E_{z}=-w \frac{\bar{E} E_{z} E_{\bar{z}}}{1+E \bar{E}} .
$$

Recall that, by Lemma $2-3, E_{z}$ have isolated zeros. Then, by a continuity argument, we deduce that the relation (2.1) in Remark 2-2 holds. Hence, by (1) of Remark 2-2, we have $\tilde{H} \equiv 1$.

We next prove that a solution of Equation (*) gives rise to a mean curvature one conformal immersion into the hyperbolic 3-space.

THEOREM 2-5. Let $U \subset C$ be a simply connected domain and $E: U \rightarrow C$ a nonholomorphic $C^{2}$ function satisfying Equation (*). Define $U^{*}=\left\{z \in U ; E_{\bar{z}}(z) \neq 0\right\}\left(U \backslash U^{*}\right.$ is discrete, see Lemma 2-3). Then there exists a map $X: U \rightarrow \boldsymbol{H}^{3}$ such that the restriction of $X$ on $U^{*}$ defines a mean curvature one conformal immersion of $U^{*}$ into $\boldsymbol{H}^{3}$ whose euclidean Gauss map is $E$. More precisely, we have

$$
\begin{gathered}
w(z)=\exp \left(-2 \operatorname{Re} \int \frac{\bar{E} E_{\bar{z}}}{1+E \bar{E}} d \bar{z}\right), \\
G(z)=\int w E_{z} d z, \\
(u+i v)(z)=(G-w E)(z) .
\end{gathered}
$$

The hyperbolic metric induced by $X$ is given by $d s=\left|E_{\bar{z}}\right||d z|$.

Furthermore, $X$ is uniquely determined up to a positive isometry of $\boldsymbol{H}^{3}$. More precisely, if $\hat{X}: U^{*} \rightarrow \boldsymbol{H}^{3}$ is another mean curvature one conformal immersion whose euclidean Gauss map is $E$, then there exists a positive real number $\lambda>0$ and a complex number $\alpha \in C$ such that

$$
\hat{X}(z)=\lambda X(z)+(\alpha, 0) .
$$

Proof. Since $U$ is simply connected and $E$ satisfies Equation (*), the proof of Lemma 2-3 shows that the real 1 -form

$$
\operatorname{Re}\left(\frac{\bar{E} E_{\bar{z}}}{1+E \bar{E}} d \bar{z}\right)
$$

is integrable. Thus we can define a strictly positive function $w$ on $U$ by setting as in (2.4). Moreover, $w$ satisfies (2.1) in Remark 2-2, which implies that $w E_{z}$ is a holomorphic function on $U$. As $U$ is simply connected, there exists a holomorphic function $G$ on $U$ as in (2.5). Now we define real functions $u$ and $v$ on $U$ by setting as in (2.6).

Let us prove that the map $X=(u, v, w): U \rightarrow \boldsymbol{H}^{3}$ restricted to $U^{*}$ is a conformal mean curvature one immersion with euclidean (resp. hyperbolic) Gauss map $E$ (resp. $G$ ). 
Observe that from (2.5) we get $G_{z}=w E_{z}$. Therefore $G_{\bar{z}}-w E_{\bar{z}}=G_{\bar{z}}-w E_{\bar{z}}+G_{z}-w E_{z}=$ $G_{x}-w E_{x}$ and $G_{\bar{z}}-w E_{\bar{z}}=G_{\bar{z}}-w E_{\bar{z}}-\left(G_{z}-w E_{z}\right)=i\left(G_{y}-w E_{y}\right)$. Thus we get $G_{y}-w E_{y}=-i\left(G_{x}-w E_{x}\right)$. Also, by using (2.4), we get

$$
w_{x}=2 \operatorname{Re}\left(\bar{E} \frac{\left(G_{x}-w E_{x}\right)}{1+E \bar{E}}\right), \quad w_{y}=2 \operatorname{Re}\left(\bar{E} \frac{\left(G_{y}-w E_{y}\right)}{1+E \bar{E}}\right) .
$$

It then follows that $X_{x} \cdot X_{x}=\left((G-w E)_{x}, w_{x}\right) \cdot\left((G-w E)_{x}, w_{x}\right)=\left|G_{x}-w E_{x}\right|^{2}$, $X_{y} \cdot X_{y}=\left|G_{y}-w E_{y}\right|^{2}=\left|G_{x}-w E_{x}\right|^{2}$ and $X_{x} \cdot X_{y}=\operatorname{Re}\left(\left(G_{x}-w E_{x}\right)\left(\bar{G}_{y}-w \bar{E}_{y}\right)\right)$. Hence we have that $X_{x} \cdot X_{x}=X_{y} \cdot X_{y}$ and $X_{x} \cdot X_{y}=0$, that is, $X$ defines a conformal immersion of $U^{*}$ into $\boldsymbol{H}^{3}$.

Now the oriented euclidean Gauss map $N$ on $U^{*}$ is given by

$$
N=\left(X_{x} \wedge X_{y}\right) /\left|X_{x} \wedge X_{y}\right| .
$$

Using the above relations, a computation shows that

$$
X_{x} \wedge X_{y}=\frac{\left|G_{x}-w E_{x}\right|^{2}}{1+E \bar{E}}(2 \operatorname{Re} E, 2 \operatorname{Im} E, E \bar{E}-1) .
$$

Thus we get on $U^{*}$

$$
N=\frac{1}{1+E \bar{E}}(2 \operatorname{Re} E, 2 \operatorname{Im} E, E \bar{E}-1)=\Pi^{-1} \circ E .
$$

We deduce that $E$ is the euclidean Gauss map of $X$ as desired.

Also from (2.6) and Proposition 1-2 we deduce that $G$ is the hyperbolic Gauss map associated to $N$. Finally, as $G$ is a holomorphic map, we conclude from Corollary 1-9 that $\tilde{H} \equiv 1$ on $U^{*}$. That is, $X: U^{*} \rightarrow \boldsymbol{H}^{3}$ is a mean curvature one conformal immersion whose induced metric is given by

$$
d s^{2}=\frac{\left|G_{\bar{z}}-w E_{\bar{z}}\right|^{2}}{w^{2}}|d z|^{2}=\left|E_{\bar{z}}\right|^{2}|d z|^{2} .
$$

This completes the proof of the existence part of the statement.

Suppose now that $\hat{X}: U^{*} \rightarrow \boldsymbol{H}^{3}$ is another conformal mean curvature one immersion with the oriented euclidean Gauss map $E$. Set $\hat{X}=(\hat{u}, \hat{v}, \hat{w})$, and consider the hyperbolic Gauss map $\hat{G}$ of $\hat{X}$. Since $\hat{G}$ is a holomorphic map (Corollary 1-9), we infer with Lemma 1-4 that the coordinate $\hat{w}$ satisfies

$$
\frac{\hat{w}_{\bar{z}}}{\hat{w}}=-\frac{\bar{E} E_{\bar{z}}}{1+E \bar{E}}=\frac{w_{\bar{z}}}{w} .
$$

This implies the existence of $\lambda>0$ such that $\hat{w}=\lambda w$. Moreover, Lemma 1-4 shows that $\hat{G}_{z}=\hat{w} E_{z}=\lambda w E_{z}=\lambda G_{z}$. Since $\hat{G}$ and $G$ are holomorphic functions, we deduce that there exists $\alpha \in C$ such that $\hat{G}=\lambda G+\alpha$. Thus

$$
\begin{aligned}
\hat{X} & =(\hat{u}+i \hat{v}, \hat{w})=(\hat{G}-\hat{w} E, \hat{w}) \\
& =(\lambda G+\alpha-\lambda w E, \lambda w)=\lambda X+(\alpha, 0) .
\end{aligned}
$$

This achieves the proof of the theorem. 
Theorem 2-5 leads immediately to a global representation:

COROLlary 2-6. Let $S$ be a Riemann surface and $E: S \rightarrow C$ a non-holomorphic $C^{2}$ map satisfying (*). Assume that

$$
\operatorname{Re} \int_{\gamma} \frac{\bar{E} E_{\bar{z}}}{1+E \bar{E}} d \bar{z}=0
$$

for every closed path $\gamma \subset S$. Let $w: S \rightarrow \boldsymbol{R}$ be a function defined by

$$
w=\exp \left(-\operatorname{Re} \int \frac{\bar{E} E_{\bar{z}}}{1+E \bar{E}} d \bar{z}\right),
$$

so that $w E_{z} d z$ is a holomorphic 1-form on $S$. Assume also that

$$
\int_{\gamma} w E_{z} d z=0
$$

for every closed path $\gamma \subset S$, that is, the 1-form $w E_{z} d z$ has a global primitive $G$ on $S$. Set $u+i v:=G-w E$.

Then the function $X:=(u, v, w): S \rightarrow \boldsymbol{H}^{3}$ defines a mean curvature one conformal immersion of $S \backslash D$ into $\boldsymbol{H}^{3}$, where $D=\left\{z \in S ; E_{\bar{z}}(z)=0\right\}$ (recall that $D$ is a discrete set, see Lemma 2-3).

We now describe in terms of $E$ the Hopf function $\tilde{\Phi}$ and the Gauss curvature $K$ of a mean curvature one surface.

Proposition 2-7. Assume that $X: U \rightarrow \boldsymbol{H}^{3}$ is a mean curvature one conformal immersion such that $E(z) \neq \infty$ for any $z \in U$. Then it holds that

$$
\tilde{\Phi}=2 \frac{E_{z}(\bar{E})_{z}}{1+E \bar{E}}, \quad K=\frac{-4}{(1+E \bar{E})^{2}} \cdot \frac{\left|E_{z}\right|^{2}}{\left|E_{\bar{z}}\right|^{2}} .
$$

The proof is a straightforward verification, by using the fact that the hyperbolic Gauss map $G$ is a holomorphic map.

REMARK 2-8. Since $U$ is simply connected, it is well-known that to each mean curvature one conformal immersion $X: U \rightarrow \boldsymbol{H}^{3}$ is associated an isometric minimal and conformal immersion $Y: U \rightarrow \boldsymbol{R}^{3}$. That is, the induced metric on $U$ by $Y$ is $d s^{2}$ and the second fundamental form of $Y$ is $\tilde{\Pi}-d s^{2}$, see [L], [B], [U-Y, 1], [SE-T, 1]. Moreover, $Y$ is uniquely determined up to a positive isometry of $\boldsymbol{R}^{3}$. Consequently, the Weierstrass representation $(g, f d z)$ associated to $X$ (namely, of the minimal immersion $Y$ associated to $X$ ) is defined up to a rotation of $\boldsymbol{R}^{3}$. Nevertheless, throughout this paper, we shall call the Weierstrass representation of any minimal immersion associated to $X$ the Weierstrass representation associated to $X$.

We deduce from Proposition 2-7 the following

COROLlaRY 2-9. Assume that $X$ is a mean curvature one conformal immersion. Let $Y: U \rightarrow \boldsymbol{R}^{3}$ be the isometric minimal immersion associated to $X$. Let $(g, f d z)$ be the 
Weierstrass representation of $Y$. Then, on $U^{*}=\{z \in U ; E(z) \neq \infty\}$, we have

$$
|f|\left(1+|g|^{2}\right)=\left|E_{\bar{z}}\right|, \quad f g_{z,}=-\frac{E_{z}(\bar{E})_{z}}{1+E \bar{E}},
$$

so that $\tilde{\Phi}=-2 f g_{z}$ on $U^{*}$. Furthermore, for each $z_{0}$ in $U$, the function $g$ and the hyperbolic Gauss map $G$ have the same order at $z_{0}$.

Proof. The metrics induced on $U$ by $X$ and $Y$ are the same, so that

$$
d s^{2}=\left|E_{\bar{z}} d z\right|^{2}=|f|^{2}\left(1+|g|^{2}\right)^{2}|d z|^{2} \text { on } U^{*} .
$$

Moreover, the Hopf functions of $X$ and $Y$ are the same. Since the Hopf function of $Y$ is $-2 f g_{z}$, it follows that

$$
\tilde{\Phi}=2 \frac{E_{z}(\bar{E})_{z}}{1+E \bar{E}}=-2 f g_{z} \text { on } U^{*} .
$$

Finally, let $z_{0}$ be any point in $U$. Up to a positive isometry of $\boldsymbol{R}^{3}$ (resp. $\boldsymbol{H}^{3}$ ) we can suppose, without changing the order of $g$ (resp. $G$ ) at $z_{0}$, that $z_{0}$ is not a pole of $g$ (resp. $G$ ), so that $E\left(z_{0}\right) \neq \infty$. Since $G_{z}=w E_{z}$ on $U^{*}$ (see Lemma 1-4), we have

$$
f g_{z}=-\frac{(\bar{E})_{z}}{w(1+E \bar{E})} \cdot G_{z} .
$$

Observe that $f$ and $(\bar{E})_{z} / w(1+E \bar{E})$ are holomorphic functions which do not vanish at $z_{0}$. Hence, $g$ has order $n \in N^{*}$ at $z_{0}$ if and only if $g_{z}$ has a zero at $z_{0}$ with multiplicity $n-1$, which is equivalent to that $G_{z}$, has a zero at $z_{0}$ with multiplicity $n-1$, that is, $G$ has order $n$ at $z_{0}$. This completes the proof of the corollary.

Remark 2-10. Assume that $\tilde{H} \equiv 1$ and $E \neq \infty$ on $U$. Then, by Lemma 1-3, one can easily verify a well-known fact that $\log w$ is a superharmonic function.

REMARK 2-11. Now we recall briefly some technical facts obtained in [SE-T, 1], since we need them to produce Examples 2-12 and 2-13. In what follows, we keep the notation of that paper.

Let $Y: U \rightarrow \boldsymbol{R}^{3}$ be a conformal minimal immersion and $(g, f d z)$ the Weierstrass representation of $Y$. Let $A, B, C$ and $D$ be holomorphic functions on $U$ given by Proposition 1-7 in [SE-T, 1]. Then, up to a positive isometry of $\boldsymbol{H}^{3}$, the associated mean curvature one immersion $X: U \rightarrow \boldsymbol{H}^{3}$ is given by

$$
\left\{\begin{array}{l}
(u+i v)(z)=\frac{\bar{A} C+\bar{B} D}{|A|^{2}+|B|^{2}}(z), \\
w(z)=\frac{1}{|A|^{2}+|B|^{2}}(z) .
\end{array}\right.
$$

As a matter of fact, $A$ and $C$ (resp. $B$ and $D$ ) are independent solutions of

$$
P^{\prime \prime}-\frac{f_{z}}{f} P^{\prime}-f g_{z} P=0(1) \quad\left(\text { resp. } Q^{\prime \prime}-\frac{\left(f g^{2}\right)_{z}}{f g^{2}} Q^{\prime}-f g_{z} Q=0 \text { (2) }\right)
$$


satisfying $A D-B C \equiv 1$ and $A_{z} D_{z}-B_{z} C_{z} \equiv 0$, see [U-Y-1, Lemma 2.1]. Observe that here we have substituted $\eta$ (in [SE-T, 1]) for $f$. Finally, the hyperbolic Gauss map $G$ of $X$ is $G=C_{z} / A_{z}$ (see Lemma 1-10 in [SE-T, 1]).

Example 2-12. The euclidean Gauss map of the catenoid-helicoid cousins.

Let us consider the Weierstrass data $(g, f d z)=\left(e^{z}, \lambda e^{i \theta} \cdot e^{-z} d z\right)$ on $U=C$, where $\lambda>0$ and $\theta \in[0,2 \pi$ [. For each positive $\lambda$, when $\theta$ varies in the interval $[0,2 \pi[$, this yields a family of isometric minimal immersions $Y_{\lambda, \theta}: \boldsymbol{C} \rightarrow \boldsymbol{R}^{3}$. In fact, this family varies continuously from the catenoids (when $e^{i \theta}= \pm 1$ ) to the helicoids (when $e^{i \theta}= \pm i$ ). Let us denote by $X_{\lambda, \theta}$ the associated mean curvature one immersion into $\boldsymbol{H}^{3}$. Let $\gamma=\gamma_{1}+i \gamma_{2}$ be a complex number such that $\gamma^{2}+\gamma-\lambda e^{i \theta}=0$. Set $A(z)=e^{\gamma z}$, and note that $A$ satisfies (2.7)-(1).

Suppose first that $1+2 \gamma \neq 0$. On account of Proposition 1-7 of [SE-T, 1], we have

$$
\left\{\begin{array}{l}
A(z)=e^{\gamma z}, \quad B(z)=-\frac{\gamma}{1+\gamma} e^{(1+\gamma) z}, \\
C(z)=-\frac{\gamma(1+\gamma)}{1+2 \gamma} e^{-(1+\gamma) z}, \quad D(z)=\frac{(1+\gamma)^{2}}{1+2 \gamma} e^{-\gamma z} .
\end{array}\right.
$$

Thus $X_{\lambda, \theta}$ is given by (see Remark 2-11)

$$
\left\{\begin{array}{l}
(u+i v)(z)=-\frac{(1+\gamma)^{2}}{1+2 \gamma} e^{-2 \gamma z} \frac{\gamma(1+\bar{\gamma}) e^{-z}+\bar{\gamma}(1+\gamma) e^{\bar{z}}}{|1+\gamma|^{2}+|\gamma|^{2} e^{z+\bar{z}}}, \\
w(z)=|1+\gamma|^{2} \frac{e^{-\gamma z-\bar{\gamma} \bar{z}}}{|1+\gamma|^{2}+|\gamma|^{2} e^{z+\bar{z}}} .
\end{array}\right.
$$

We deduce also that

$$
G=\frac{C_{z}}{A_{z}}=\frac{(1+\gamma)^{2}}{1+2 \gamma} e^{-(1+2 \gamma) z} .
$$

Performing some calculations combined with Proposition 1-2, we get

$$
E=e^{\overline{\gamma z}-\gamma z}\left((1+\gamma) e^{-z}+\bar{\gamma} \cdot \frac{1+\gamma}{1+\bar{\gamma}} e^{\bar{z}}\right) .
$$

Note that for any $y_{0} \in \boldsymbol{R}$ we have

$$
\begin{aligned}
(u+i v)\left(z+i y_{0}\right) & =e^{-i y_{0}(1+2 \operatorname{Re} \gamma)} \cdot e^{2 y_{0} \operatorname{Im} \gamma}(u+i v)(z), \\
w\left(z+i y_{0}\right) & =e^{2 y_{0} \operatorname{In} \gamma} w(z) .
\end{aligned}
$$

Suppose now that $1+2 \gamma=0$ (that is, $\lambda=1 / 4$ and $e^{i \theta}=-1$ ). Using Proposition 1-7 of [SE-T, 1], we get $B(z)=e^{z / 2}, C(z)=(-z / 4) e^{-z / 2}$ and $D(z)=(1-z / 4) e^{z / 2}$. We infer 
that

$$
\left\{\begin{array}{l}
(u+i v)(z)=\frac{e^{(z+\bar{z}) / 2}}{e^{(z+\bar{z}) / 2}+e^{-(z+\bar{z}) / 2}}-\frac{z}{4} \\
w(z)=\frac{1}{e^{(z+\bar{z}) / 2}+e^{-(z+\bar{z}) / 2}} \\
G(z)=\frac{C_{z}}{A_{z}}=-\frac{1}{4}(z-2)
\end{array}\right.
$$

Thus

$$
E(z)=\frac{1}{2} e^{-z / 2} e^{\bar{z} / 2}\left(-e^{z}+e^{-\bar{z}}\right) .
$$

We remark that $w\left(z+i y_{0}\right)=w(z)$ and $(u+i v)\left(z+i y_{0}\right)=(u+i v)(z)-i y_{0} / 4$ for any $y_{0} \in \boldsymbol{R}$.

We then deduce the following:

(i) When $e^{i \theta}=1$, or $e^{i \theta}=-1$ and $\lambda<1 / 4$ (that is, $\operatorname{Im} \gamma=0$ and $1+2 \gamma \neq 0$ ), $X_{\lambda, \theta}$ is a rotational immersion, called catenoid cousin by Bryant, see [B].

(ii) When $e^{i \theta}=-1$ and $\lambda=1 / 4$ (that is, $1+2 \gamma=0$ ), the associated surface (see figure 0-a) is invariant under the euclidean horizontal translations in $\boldsymbol{H}^{3}$ :

$$
(u, v, w) \rightarrow(u, v, w)+\left(0, y_{0}, 0\right) \text { for any } y_{0} \in \boldsymbol{R} .
$$

The profile curve, called "courbe des forçats" (see figure 0-b), was studied by Poleni in 1729, see [Rev]. This surface is known as a dual of Enneper's cousin, see [R-U-Y, 3].

(iii) When $e^{i \theta}=-1$ and $\lambda>1 / 4$ (that is, $1+2 \operatorname{Re} \gamma=0$ and $\operatorname{Im} \gamma \neq 0$ ), $X_{\lambda, \theta}$ is invariant under a one-parameter group of hyperbolic translations:

$$
(u, v, w) \rightarrow \lambda \cdot(u, v, w) \text { for any } \lambda>0 .
$$

(iv) In all other cases (that is, $e^{i \theta} \neq \pm 1$, or equivalently $\operatorname{Im} \gamma \neq 0$ and $1+2 \operatorname{Re} \gamma \neq 0$ ), $X_{\lambda, \theta}$ is a non-trivial helicoidal immersion.

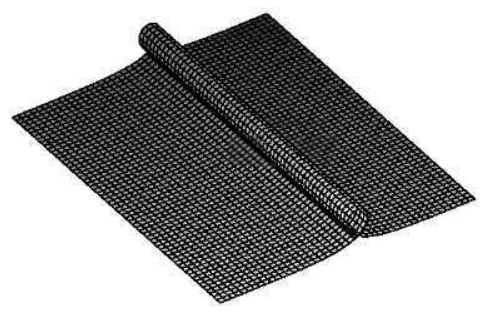

FIGURE 0-a.

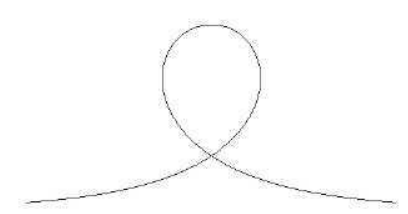

FIGURE 0-b.

Now, let us show that if $\operatorname{Im} \gamma \neq 0$ (that is, when the immersion is neither a rotational one nor invariant by an euclidean horizontal translation), then the asymptotic boundary of the 
surface is the whole $\partial_{\infty} \boldsymbol{H}^{3}$. Indeed, by setting $z=x+i y$ and $\gamma=\gamma_{1}+i \gamma_{2}$, we have

$$
\left\{\begin{aligned}
(u+i v)(z)=- & \frac{(1+\gamma)^{2}}{1+2 \gamma} \cdot e^{2 \gamma_{2} y-x\left(1+2 \gamma_{1}\right)} \cdot e^{-i\left(y\left(1+2 \gamma_{1}\right)+2 \gamma_{2} x\right)} \\
& \cdot \frac{\bar{\gamma}(1+\gamma) e^{x}+\gamma(1+\bar{\gamma}) e^{-x}}{|1+\gamma|^{2} e^{-x}+|\gamma|^{2} e^{x}} \\
w(z)=|1+\gamma|^{2} & \frac{e^{2 \gamma_{2} y-x\left(1+2 \gamma_{1}\right)}}{|1+\gamma|^{2} e^{-x}+|\gamma|^{2} e^{x}} .
\end{aligned}\right.
$$

Let $u_{0}+i v_{0} \in \boldsymbol{C}^{*}$ be any non-zero complex number and $\alpha \in \boldsymbol{R}$ such that

$$
e^{\alpha}\left|\frac{(1+\gamma)^{2}}{1+2 \gamma}\right| \cdot\left|\frac{1+\gamma}{\gamma}\right|=\left|u_{0}+i v_{0}\right| \text {. }
$$

Denote by $z_{\alpha}=x+i y$ the complex numbers such that $2 \gamma_{2} y-x\left(1+2 \gamma_{1}\right)=\alpha$. We then have

$$
\lim _{x \rightarrow \pm \infty} w\left(z_{\alpha}\right)=0, \quad \lim _{x \rightarrow+\infty}\left|(u+i v)\left(z_{\alpha}\right)\right|=\left|u_{0}+i v_{0}\right|
$$

Furthermore, as $y\left(1+2 \gamma_{1}\right)+2 \gamma_{2} x=\left(x\left(\left(1+2 \gamma_{1}\right)^{2}+4 \gamma_{2}^{2}\right)+\alpha\left(1+2 \gamma_{1}\right)\right) / 2 \gamma_{2}$, it is easy to see that $\operatorname{Arg}(u+i v)\left(z_{\alpha}\right)$ approaches any real number, modulo $2 \pi$, when $x \rightarrow+\infty$. Consequently, there exists a subsequence $z_{\alpha, n}=x_{n}+i y_{n}$ such that $\lim _{n \rightarrow+\infty} x_{n}=+\infty$ and $\lim \operatorname{Arg}(u+$ iv) $\left(z_{\alpha, n}\right)=\operatorname{Arg}\left(u_{0}+i v_{0}\right)$. We infer that $u_{0}+i v_{0}$ lies on the asymptotic boundary of the surface. Since this is true for any non-zero complex number $u_{0}+i v_{0}$, we conclude that the asymptotic boundary is the whole $\partial_{\infty} \boldsymbol{H}^{3}$ as claimed.

It can be easily inferred that in the case where $\gamma \in \boldsymbol{R}-\{-1 / 2\}$ (that is, when the immersion is rotational), the asymptotic boundary is $\{0, \infty\}$. Finally, if $\gamma=-1 / 2$ (that is, when the immersion is invariant under euclidean horizontal translations), then the asymptotic boundary is $\{\infty\}$.

EXAMPLE 2-13. The euclidean Gauss map of the Enneper cousins.

Let us now consider the Weierstrass representation of Enneper surfaces on $U=C$ : $(g(z), f(z) d z)=(z, \alpha d z)$, where $\alpha \in C^{*}$. The equations (2.7) in Remark 2-11 are

$$
P^{\prime \prime}-\alpha P=0(1) \text { and } Q^{\prime \prime}-\frac{2}{z} Q^{\prime}-\alpha Q=0
$$

Let $\gamma \in C$ be any square root of $\alpha$, that is, $\gamma^{2}=\alpha$. Then the function $A(z)=e^{\gamma z}$ is a solution of (1). Thus, taking into account of Proposition 1-7 of [SE-T, 1], we get

$$
B(z)=\left(-z+\frac{1}{\gamma}\right) e^{\gamma z}, \quad C(z)=-\frac{\gamma}{2} e^{-\gamma z}, \quad D(z)=\frac{1}{2}(\gamma z+1) e^{-\gamma z},
$$

from which we deduce that

$$
\left\{\begin{array}{l}
(u+i v)(z)=\frac{\left(-|\gamma|^{2}+(-\overline{\gamma z}+1)(\gamma z+1)\right) e^{-2 \gamma z}}{2 \bar{\gamma}\left(1+|z-1 / \gamma|^{2}\right)} \\
w(z)=\frac{1}{\left(1+|z-1 / \gamma|^{2}\right) e^{\gamma z+\overline{\gamma z}}}
\end{array}\right.
$$


Moreover, $G(z)=\left(C_{z} / A_{z}\right)(z)=\left(\gamma e^{-2 \gamma z}\right) / 2$. Therefore, using Proposition 1-2, we get

$$
E(z)=\frac{\gamma}{\bar{\gamma}} z e^{-\gamma z} e^{\overline{\gamma z}}\left(\frac{\bar{\gamma}}{z}-1+\overline{\gamma z}\right) \text {. }
$$

3. Meromorphic data. Recall that $H$ (resp. $\tilde{H}$ ) denotes the euclidean (resp. hyperbolic) mean curvature with respect to $N$ (resp. $\tilde{N}$ ). Furthermore, $U \subset C$ is a simply connected domain.

Remark 3-1. Assume that $\tilde{H} \equiv 1$ and $E_{z} \equiv 0$. Then we see that $X$ is an euclidean totally umbilic immersion, so that it is a hyperbolic totally umbilic immersion. Hence we may conclude that $X(U)$ is part of a horosphere.

Throughout this section we consider the equation

$$
E_{z \bar{z}}=\frac{\bar{E}}{1+E \bar{E}} E_{z} E_{\bar{z}},
$$

where $E: U \rightarrow C \cup\{\infty\}$ is a $C^{2}$ map. In fact, we require that (*) holds on $U-\{z \in$ $U ; E\left(z_{0}\right)=\infty$ \}. When $E$ is the oriented euclidean Gauss map of a mean curvature one conformal immersion $X: U \rightarrow \boldsymbol{H}^{3}$, the set $\left\{z \in U ; E\left(z_{0}\right)=\infty\right\}$ is discrete unless $X$ is a totally umbilic immersion (see Lemma 2-3). When $E$ is the euclidean Gauss map of a conformal immersion $X$, we have seen in Section 2 that $(*)$ is a necessary condition to have $\tilde{H} \equiv 1$ (see Proposition 2-1).

Definition 3-2. Let $E: U \rightarrow C \cup\{\infty\}$ be a $C^{2}$ function. We say that $E$ is a non-trivial solution of Equation (*) if $E$ is a solution of $(*)$ and is neither meromorphic nor anti-meromorphic. Otherwise, we say that $E$ is a trivial solution of Equation (*). This terminology is justified by Remark 3-1.

LEMma 3-3. Let E be a solution of (*) which has one of the forms

$$
E=I \bar{J} \text { or } E=I+\bar{J},
$$

where $I$ and $J$ are meromorphic functions on $U$. Then $E$ is a trivial solution of $(*)$.

PRooF. Suppose that $E=I \bar{J}$. We have $E_{z}=I_{z} \bar{J}, E_{\bar{z}}=I \overline{J_{z}}$ and $E_{z \bar{z}}=I_{z} \overline{J_{z}}$. Thus (*) holds if and only if $I_{z} \overline{J_{z}}=0$. This implies $I_{z} \equiv 0$ or $J_{z} \equiv 0$. Then $E$ is either antimeromorphic or else $E$ is meromorphic. The case where $E=I+\bar{J}$ can be treated in the same way.

We observe that Examples 2-12 and 2-13 suggest to look for solutions of $(*)$ of the following form

$$
E=h \bar{S}(T+\bar{R}),
$$

where $h, R, S$ and $T$ are meromorphic functions on $U$. As a matter of fact, the form (3.1) is invariant under positive isometry of $\boldsymbol{H}^{3}$ (see Proposition 3-7) and, surprisingly, we shall see that every solution of $(*)$ must have this form (see Theorem 3-13). 
LEMMA 3-4. Let $E: U \rightarrow C \cup\{\infty\}$ be a solution of $(*)$ of the form (3.1). If one of the functions $h, R, S$ or $T$ is constant, then $E$ is a trivial solution of (*).

PROOF. If $T$ or $R$ is constant, then we already know from Lemma 3-3 that $E$ is a trivial solution of $(*)$.

Suppose that $h$ is constant and set $h \equiv c \in C^{*}$. We then have $E=c \bar{S}(T+\bar{R})$, $E_{z}=c \bar{S} T_{z}, E_{\bar{z}}=c \overline{S_{z}}(T+\bar{R})+c \overline{S R_{z}}$ and $E_{z \bar{z}}=c \overline{S_{z}} T_{z}$. Note that if $S$ were also constant, then $E$ should be a trivial solution of (*). Hence, let us suppose that $S$ is not constant on $U$. Thus (*) holds if and only if

$$
\frac{1}{c \bar{c} S}=R \overline{\left(\frac{S^{2} R_{z}}{S_{z}}\right)}+\overline{\left(\frac{T S^{2} R_{z}}{S_{z}}\right)}
$$

Differentiating this with respect to $\bar{z}$, we have

$$
R \overline{\left(\frac{S^{2} R_{z}}{S_{z}}\right)_{z}}+\overline{\left(\frac{T S^{2} R_{z}}{S_{z}}\right)_{z}}=0
$$

Hence $R$ is constant or $\overline{\left(S^{2} R_{z} / S_{z}\right)_{z}}=\overline{\left(T S^{2} R_{z} / S_{z}\right)_{z}}=0$.

Therefore, if $R$ is not constant, we infer easily that $T$ is constant. In each case we see that $E$ must be a trivial solution of (*). We can apply the same argument when $S$ is constant. This achieves the proof of the lemma.

We are looking for non-trivial solutions of Equation (*).

Proposition 3-5. Let $E: U \rightarrow C \cup\{\infty\}$ be a $C^{2}$ function of the form (3.1). Then $E$ is a non-trivial solution of $(*)$ if and only if there exist two complex numbers $a$ and $b, a \neq 0$, such that

$$
\left\{\begin{array}{l}
S=\frac{(b+a T) h_{z}+a h T_{z}}{h^{2} T_{z}}, \\
R=\frac{\bar{b}}{\bar{a}}+\frac{1}{\bar{a}} \cdot \frac{h_{z}}{(b+a T) h_{z}+a h T_{z}}, \\
h \neq \frac{1}{\alpha T+\beta} \text { for any complex numbers } \alpha, \beta .
\end{array}\right.
$$

Consequently, we have

$$
E=\frac{h}{a} \cdot \overline{\left(\frac{(b+a T) h_{z}+a h T_{z}}{h^{2} T_{z}}\right)}\left(b+a T+\overline{\left(\frac{h_{z}}{(b+a T) h_{z}+a h T_{z}}\right)}\right) .
$$

Conversely, for any meromorphic functions $h$ and $T$ on $U$ and for any complex numbers $a$ and $b, a \neq 0$, the function $E=h \bar{S}(T+\bar{R})$, where $R$ and $S$ are defined as above, is a solution of $(*)$. 
PROOF. Let $E=h \bar{S}(T+\bar{R})$ be a non-trivial solution of (*). Consequently, $h, R, S$ and $T$ are non-constant meromorphic functions on $U$. Therefore $(*)$ is written as

$$
\left[\overline{R S_{z}+S R_{z}}\right]+\overline{S_{z}}\left(\frac{T h_{z}+h T_{z}}{h_{z}}\right)=\left(\frac{h^{2} S T_{z}}{h_{z}}\right) \overline{h S^{2} T R_{z}}+\left(\frac{h^{2} S R T_{z}}{h_{z}}\right) \overline{h S^{2} R_{z}} .
$$

Taking the derivative of the last equality with respect to $z$, we get

$$
\overline{S_{z}}\left(\frac{T h_{z}+h T_{z}}{h_{z}}\right)_{z}=\left(\frac{h^{2} S T_{z}}{h_{z}}\right)_{z} \overline{h S^{2} T R_{z}}+\left(\frac{h^{2} S R T_{z}}{h_{z}}\right)_{z} \overline{h S^{2} R_{z}} .
$$

Observe that if $\left(\left(T h_{z}+h T_{z}\right) / h_{z}\right)_{z} \equiv 0$, then we should have one of the following possibilities:

$$
\text { (1) } \frac{\overline{h S^{2} T R_{z}}}{\overline{h S^{2} R_{z}}} \text { is constant, or else (2) }\left(\frac{h^{2} S T_{z}}{h_{z}}\right)_{z}=\left(\frac{h^{2} S R T_{z}}{h_{z}}\right)_{z}=0 \text {. }
$$

If the case (1) occurs, we infer that $T$ is constant. Furthermore, if the case (2) occurs, then $h^{2} S T_{z} / h_{z}$ and $h^{2} S R T_{z} / h_{z}$ are constant, so that $R$ is constant. Therefore both cases contradict the assumption that $E$ is non-trivial. Hence $\left(\left(T h_{z}+h T_{z}\right) / h_{z}\right)_{z} \not \equiv 0$. Thus (*) implies

$$
\overline{S_{z}}=\frac{\left(h^{2} S T_{z} / h_{z}\right)_{z}}{\left(\left(T h_{z}+h T_{z}\right) / h_{z}\right)_{z}} \overline{h S^{2} T R_{z}}+\frac{\left(h^{2} S R T_{z} / h_{z}\right)_{z}}{\left(\left(T h_{z}+h T_{z}\right) / h_{z}\right)_{z}} \overline{h S^{2} R_{z}} .
$$

Differentiating this by $z$, we then get

$$
0=\left(\frac{\left(h^{2} S T_{z} / h_{z}\right)_{z}}{\left(\left(T h_{z}+h T_{z}\right) / h_{z}\right)_{z}}\right)_{z} \overline{h S^{2} T R_{z}}+\left(\frac{\left(h^{2} S R T_{z} / h_{z}\right)_{z}}{\left(\left(T h_{z}+h T_{z}\right) / h_{z}\right)_{z}}\right)_{z} \overline{h S^{2} R_{z}} .
$$

Since $T$ is not a constant function, it follows that

$$
\left(\frac{\left(h^{2} S T_{z} / h_{z}\right)_{z}}{\left(\left(T h_{z}+h T_{z}\right) / h_{z}\right)_{z}}\right)_{z}=0 \text { and }\left(\frac{\left(h^{2} S R T_{z} / h_{z}\right)_{z}}{\left(\left(T h_{z}+h T_{z}\right) / h_{z}\right)_{z}}\right)_{z}=0 .
$$

Consequently, there exist complex numbers $a, b, c$ and $d$ such that

$$
\left\{\begin{array}{l}
\frac{h^{2} S T_{z}}{h_{z}}=a \frac{T h_{z}+h T_{z}}{h_{z}}+b, \\
\frac{h^{2} S R T_{z}}{h_{z}}=c \frac{T h_{z}+h T_{z}}{h_{z}}+d .
\end{array}\right.
$$

Hence we get

$$
\left\{\begin{array}{l}
S=\frac{(b+a T) h_{z}+a h T_{z}}{h^{2} T_{z}}, \\
R=\frac{(d+c T) h_{z}+c h T_{z}}{(b+a T) h_{z}+a h T_{z}} .
\end{array}\right.
$$

Note that we must have $a d-b c \neq 0$, otherwise $R$ should be constant. 
Now, we are going to look for the necessary and sufficient condition about $a, b, c$ and $d$ so that $E=h \bar{S}(T+\bar{R})$ is a non-trivial solution of (*), where $R$ and $S$ are given by (3.3). For this purpose, assume that $S$ and $R$ satisfy (3.3). We infer that (3.2) is written as

$$
\begin{aligned}
& \left.\overline{\left(h T_{z} h_{z z}-2 h_{z}^{2} T_{z}-h h_{z} T_{z z}\right.}\right) \cdot\left(h_{z} \overline{\left(\frac{(d+c T)}{h^{3} T_{z}^{2}}\right)}+\left(T h_{z}+h T_{z}\right) \overline{\left(\frac{(b+a T)}{h^{3} T_{z}^{2}}\right)}\right) \\
& =\left(\overline { h T _ { z } h _ { z z } - 2 h _ { z } ^ { 2 } T _ { z } - h h _ { z } T _ { z z } ) } \cdot \left(\left((b+a T) h_{z}+a h T_{z}\right) \overline{\left(\frac{h T(a d-b c)}{h^{4} T_{z}^{2}}\right)}\right.\right. \\
& \left.+\left((d+c T) h_{z}+c h T_{z}\right) \cdot \overline{\left(\frac{(a d-b c) h}{h^{4} T_{z}^{2}}\right)}\right) .
\end{aligned}
$$

Observe that $\left(h T_{z} h_{z z}-2 h_{z}^{2} T_{z}-h h_{z} T_{z z}\right) \equiv 0$ implies that $R$ and $S$ are constant functions, a contradiction. On the other hand, $\left(h T_{z} h_{z z}-2 h_{z}^{2} T_{z}-h h_{z} T_{z z}\right)=0$ if and only if $h_{z z} / h_{z}-$ $2 h_{z} / h=T_{z z} / T_{z}$, or equivalently $h=-1 /\left(e^{\alpha_{1}} T+\alpha_{2}\right)$ for certain complex numbers $\alpha_{1}$ and $\alpha_{2}$. Thus $h$ cannot have the form $h=1 /(\alpha T+\beta)$

Since $\left(h T_{z} h_{z z}-2 h_{z}^{2} T_{z}-h h_{z} T_{z z}\right) \neq 0$, we have

$$
\begin{aligned}
(\bar{d}-d(\overline{a d-b c})) h_{z} & +(\bar{b}-c(\overline{a d-b c}))\left(T h_{z}+h T_{z}\right) \\
& =\left[(b(\overline{a d-b c})-\bar{c}) h_{z}+(a(\overline{a d-b c})-\bar{a})\left(T h_{z}+h T_{z}\right)\right] \cdot \bar{T} .
\end{aligned}
$$

Since $\bar{T}$ is a non-constant anti-holomorphic function, we may deduce

$$
\left\{\begin{array}{l}
(\bar{d}-d(\overline{a d-b c})) h_{z}+(\bar{b}-c(\overline{a d-b c}))\left(T h_{z}+h T_{z}\right)=0, \\
\left.(b(\overline{a d-b c})-\bar{c}) h_{z}+(\overline{a(a d-b c})-\bar{a}\right)\left(T h_{z}+h T_{z}\right)=0 .
\end{array}\right.
$$

But it is easily seen that if $h$ and $T$ satisfy the relation $\alpha_{1} h_{z}+\alpha_{2}\left(h T_{z}+T h_{z}\right)=0$ with complex numbers $\alpha_{1}, \alpha_{2}$ such that $\alpha_{1} \neq 0$ or $\alpha_{2} \neq 0$, then $h=\alpha_{3} /\left(\alpha_{2} T+\alpha_{1}\right)$ for some complex number $\alpha_{3}$. This implies that $E$ is a trivial solution of $(*)$. Thus we must have

$$
\begin{cases}\bar{d}-d(\overline{a d-b c})=0, & \bar{b}-c(\overline{a d-b c})=0 \\ b(\overline{a d-b c})-\bar{c}=0, & a(\overline{a d-b c})-\bar{a}=0 .\end{cases}
$$

It is easy to see that $a \neq 0, d \neq 0$ and $a d-b c \neq 0$. Thus (3.4) leads to

$$
c=a \bar{b} / \bar{a}, \quad d=(1+b \bar{b}) / \bar{a} .
$$

Putting these relations into the expression of $R$, we conclude as promised that

$$
R=\frac{\bar{b}}{\bar{a}}+\frac{1}{\bar{a}} \cdot \frac{h_{z}}{(b+a T) h_{z}+a h T_{z}} .
$$

Finally, let us consider any meromorphic functions $h$ and $T$ on $U$. Let $a$ and $b$ be any complex numbers, $a \neq 0$. Set $E=h \bar{S}(T+\bar{R})$, where $R$ and $S$ are defined as in the statement. Then the above proof shows that $E$ is a solution of $(*)$.

We shall need the following propositions. 
Proposition 3-6. Assume that $\tilde{H} \equiv 1$ and $E: U \rightarrow C \cup\{\infty\}$ has the form as in Proposition 3-5. Then, up to a multiplicative positive constant, we have

$$
w=\frac{\left|h^{2} T_{z}\right|^{2}}{\left|(b+a T) h_{z}+a h T_{z}\right|^{2}+\left|h_{z}\right|^{2}}, \quad G_{z}=\frac{1}{a} h^{2} T_{z} .
$$

Furthermore, the induced metric and the Hopf function are given respectively by

$$
\begin{gathered}
d s^{2}=\left|E_{\bar{z}} d z\right|^{2}=\frac{\left|h T_{z} h_{z z}-2 h_{z}^{2} T_{z}-h h_{z} T_{z z}\right|^{2}}{|a|^{2}\left|h T_{z}\right|^{4}}\left(1+|b+a T|^{2}\right)^{2}|d z|^{2}, \\
\tilde{\Phi}=2 \frac{h T_{z} h_{z z}-2 h_{z}^{2} T_{z}-h h_{z} T_{z z}}{h^{2} T_{z}} .
\end{gathered}
$$

Proof. We deduce from Proposition 3-5 that

$$
E_{z}=\frac{1}{a \overline{h^{2} T_{z}}}\left(\left|(b+a T) h_{z}+a h T_{z}\right|^{2}+\left|h_{z}\right|^{2}\right) .
$$

If we set

$$
t(z)=\frac{\left|h^{2} T_{z}\right|^{2}}{\left|(b+a T) h_{z}+a h T_{z}\right|^{2}+\left|h_{z}\right|^{2}},
$$

then $t \cdot E_{z}=(1 / a) h^{2} T_{z}$. Therefore, $t$ is a real function such that $t \cdot E_{z}$ is meromorphic on $U$. Notice that $t$ is a unique real function, up to a multiplicative constant, with this property. Since $\tilde{H}=1$, we have that $w E_{z}=G_{z}$ is a meromorphic function. Hence, up to a multiplicative constant, we have

$$
w(z)=\frac{\left|h^{2} T_{z}\right|^{2}}{\left|(b+a T) h_{z}+a h T_{z}\right|^{2}+\left|h_{z}\right|^{2}}, \quad G_{z}=w E_{z}=\frac{1}{a} h^{2} T_{z} .
$$

Furthermore, we deduce from Proposition 3-5

$$
E_{\bar{z}}=h \frac{\overline{h T_{z} h_{z z}-2 h_{z}^{2} T_{z}-h h_{z} T_{z z}}}{a \bar{h}^{3} \bar{T}_{z}^{2}}\left(1+|b+a T|^{2}\right) .
$$

Hence we have

$$
d s^{2}=\left|E_{\bar{z}} d z\right|^{2}=\frac{\left|h T_{z} h_{z z}-2 h_{z}^{2} T_{z}-h h_{z} T_{z z}\right|^{2}}{|a|^{2}\left|h T_{z}\right|^{4}}\left(1+|b+a T|^{2}\right)^{2}|d z|^{2} .
$$

By a straightforward calculation with Proposition 2-7 and 3-5, we easily infer that

$$
\tilde{\Phi}=2 \frac{h T_{z} h_{z z}-2 h_{z}^{2} T_{z}-h h_{z} T_{z z}}{h^{2} T_{z}} .
$$

Proposition 3-7. Assume that $\tilde{H} \equiv 1$ and $E$ has the form (3.1). Let $J: \boldsymbol{H}^{3} \rightarrow \boldsymbol{H}^{3}$ be a positive isometry of $\boldsymbol{H}^{3}$ and $\hat{E}$ the euclidean oriented Gauss map of the immersion $J \circ X$. Then $\hat{E}$ has also the form (3.1).

The proof, based on Lemma 1-8, is a straightforward computation.

We shall give the Weierstrass representation $(g, f d z)$ of the associated minimal immersion in $\boldsymbol{R}^{3}$ in the case where $E$ has the form (3.1). 
Proposition 3-8. Assume that $\tilde{H} \equiv 1$ and $E: U \rightarrow C \cup\{\infty\}$ has the form as in Proposition 3-5. Let $(g, f d z)$ be the Weierstrass representation of the isometric minimal immersion in $\boldsymbol{R}^{3}$ associated to $X$ (see Remark 2-8-(3)). Then we have

$$
g=b+a T, \quad f d z=-\frac{1}{a T_{z}} \cdot \frac{h T_{z} h_{z z}-2 h_{z}^{2} T_{z}-h h_{z} T_{z z}}{h^{2} T_{z}} d z .
$$

PROOF. We define holomorphic functions $\hat{g}$ and $\hat{f}$ on $U$ by

$$
\hat{g}=b+a T, \quad \hat{f}=-\frac{1}{a T_{z}} \cdot \frac{h T_{z} h_{z z}-2 h_{z}^{2} T_{z}-h h_{z} T_{z z}}{h^{2} T_{z}} .
$$

Then the data $(\hat{g}, \hat{f} d z)$ define a minimal immersion $\hat{Y}$ of $U$ into $\boldsymbol{R}^{3}$ with induced metric $d s^{2}=\left|E_{\bar{z}}\right|^{2}|d z|^{2}$ and the Hopf function $\tilde{\Phi}=2 E_{z}(\bar{E})_{z} /(1+E \bar{E})$. Then the associated mean curvature one immersion $\hat{X}$ has the same induced metric and the Hopf function as those of $X$. We deduce that $X$ and $\hat{X}$ differ by a positive isometry of $\boldsymbol{H}^{3}$. Consequently, they have the same associated minimal immersion in $\boldsymbol{R}^{3}$. We conclude therefore that we can choose $g=\hat{g}$ and $f=\hat{f}$. This completes the proof of the proposition.

We now give a meromorphic data for a mean curvature one conformal immersion in $\boldsymbol{H}^{3}$.

THEOREM 3-9 (Existence of Meromorphic Data). Let $U \subset C$ be a simply connected domain and $X: U \rightarrow \boldsymbol{H}^{3}$ a non-totally umbilic conformal immersion. Let $E$ be the oriented euclidean Gauss map of $X$. Assume that $X$ has mean curvature one with respect to $E$ (therefore, Equation (*) as in Proposition 2-1). Then there exist meromorphic functions $h, T$ on $U$ such that

$$
E=h \cdot \overline{\left(\frac{T h_{z}+h T_{z}}{h^{2} T_{z}}\right)}\left(T+\overline{\left(\frac{h_{z}}{T h_{z}+h T_{z}}\right)}\right) .
$$

Furthermore, up to a multiplicative positive constant, we have

$$
\begin{gathered}
w=\frac{\left|h^{2} T_{z}\right|^{2}}{\left|T h_{z}+h T_{z}\right|^{2}+\left|h_{z}\right|^{2}}, \\
G_{z}=h^{2} T_{z} .
\end{gathered}
$$

Up to the same multiplicative positive constant and an additive complex constant, we also have

$$
u+i v=G-\frac{h^{3} T_{z}}{\left|T h_{z}+h T_{z}\right|^{2}+\left|h_{z}\right|^{2}} \cdot \overline{\left(T h_{z}+h T_{z}\right)} \cdot\left(T+\overline{\left(\frac{h_{z}}{T h_{z}+h T_{z}}\right)}\right) .
$$

Moreover, we obtain

$$
\begin{gathered}
d s^{2}=\left|E_{\bar{z}} d z\right|^{2}=\frac{\left|h T_{z} h_{z z}-2 h_{z}^{2} T_{z}-h h_{z} T_{z z}\right|^{2}}{\left|h T_{z}\right|^{4}}\left(1+|T|^{2}\right)^{2}|d z|^{2}, \\
\tilde{\Phi}=2 \frac{h T_{z} h_{z z}-2 h_{z}^{2} T_{z}-h h_{z} T_{z z}}{h^{2} T_{z}},
\end{gathered}
$$




$$
\begin{gathered}
\tilde{\Pi}=\operatorname{Re}\left(\tilde{\Phi}(d z)^{2}\right)+d s^{2}, \\
K=-4 \frac{|h|^{4}\left|T_{z}\right|^{6}}{\left|h T_{z} h_{z z}-2 h_{z}^{2} T_{z}-h h_{z} T_{z z}\right|^{2}\left(1+|T|^{2}\right)^{4}},
\end{gathered}
$$

and, up to a rigid motion of $\boldsymbol{R}^{3}$, we can choose

$$
\begin{gathered}
g=T, \\
f=-\frac{1}{T_{z}} \cdot \frac{h T_{z} h_{z z}-2 h_{z}^{2} T_{z}-h h_{z} T_{z z}}{h^{2} T_{z}},
\end{gathered}
$$

where $(g, f d z)$ is the Weierstrass representation of the minimal immersion in $\boldsymbol{R}^{3}$ associated to $X$.

Proof. Let $Y: U \rightarrow R^{3}$ be the minimal conformal immersion associated to $X$ and $(g, f d z)$ the Weierstrass representation of $Y$. In order to prove the relation (3.5), we look for a meromorphic function $\hat{h}$ on $U$ satisfying

$$
f=-\frac{\hat{h} g_{z} \hat{h}_{z z}-2 \hat{h}_{z}^{2} g_{z}-\hat{h} \hat{h}_{z} g_{z z}}{\hat{h}^{2} g_{z}^{2}}
$$

so that the map $\hat{E}$ on $U$ defined by

$$
\hat{E}=\hat{h} \cdot \overline{\left(\frac{g \hat{h}_{z}+\hat{h} g_{z}}{\hat{h}^{2} g_{z}}\right)}\left(g+\overline{\left(\frac{\hat{h}_{z}}{g \hat{h}_{z}+\hat{h} g_{z}}\right)}\right)
$$

is the euclidean Gauss map of a mean curvature one conformal immersion $\hat{X}:=(\hat{u}, \hat{v}, \hat{w})$ : $U \rightarrow \boldsymbol{H}^{3}$ such that $X$ and $\hat{X}$ share the same metric and the same second fundamental form.

Assume first that such $\hat{h}$ and $\hat{X}$ exist. Consequently, $X$ and $\hat{X}$ differ by a positive isometry of $\boldsymbol{H}^{3}$. Therefore, since $\hat{E}$ has the form (3.1), we may conclude with the aid of Proposition 3-7 that $E$ has also the form (3.1) on $U$. Thus we deduce from Proposition 3-5 that there exist two meromorphic functions $I$ and $J$ on $U$ and two complex numbers $a$ and $b, a \neq 0$, such that

$$
E=\frac{I}{a} \cdot \overline{\left(\frac{(b+a J) I_{z}+a I J_{z}}{I^{2} J_{z}}\right)}\left(b+a J+\overline{\left(\frac{I_{z}}{(b+a J) I_{z}+a I J_{z}}\right)}\right)
$$

on $U$. Now, setting $h=I / a$ and $T=b+a J$, we have

$$
E=h \cdot \overline{\left(\frac{T h_{z}+h T_{z}}{h^{2} T_{z}}\right)}\left(T+\overline{\left(\frac{h_{z}}{T h_{z}+h T_{z}}\right)}\right)
$$

as desired. Thus, to prove the relation (3.5), it remains to show that the equation (3.15) admits a meromorphic solution $\hat{h}$ on $U$ and that there exists a mean curvature one conformal immersion $\hat{X}: U \rightarrow \boldsymbol{H}^{3}$ satisfying the conditions stated above.

For this purpose, we choose a point $z_{0} \in U$ such that $g\left(z_{0}\right) \neq \infty$ and $g_{z}\left(z_{0}\right) \neq 0$. Set $\varphi=\hat{h}_{z} / \hat{h}$. Then (3.15) holds if and only if

$$
\varphi_{z}-\varphi^{2}-\varphi \frac{g_{z z}}{g_{z}}+f g_{z}=0 .
$$


Since $g_{z z} / g_{z}$ is a holomorphic function (without pole) in a neighborhood of $z_{0}$, the equation (3.16) (with respect to $\varphi$ ) is regular at $z=z_{0}$. Therefore, (3.16) can be integrated in a neighborhood of $z_{0}$. Thus we get a holomorphic solution $\varphi$ of (3.16) defined on a simply connected neighborhood $\hat{U} \subset U$ of $z_{0}$. Hence, using the relation $\varphi=\hat{h}_{z} / \hat{h}$, we get a holomorphic solution $\hat{h}$ of (3.15) defined on $\hat{U}$. We shall show that $\hat{h}$ can be extended to a meromorphic function on $U$.

Since $\hat{h}\left(z_{0}\right) \neq 0$, we can suppose that $\hat{h} \neq 0$ on $\hat{U}$. Moreover, we can assume that $g$ is a holomorphic map such that $g_{z} \neq 0$ on $\hat{U}$. Consider the function $\hat{E}$ on $\hat{U}$ which has the form (3.1), defined by $\hat{h}$ and $g$ with complex constants $a=1$ and $b=0$. Namely,

$$
\hat{E}(z)=\hat{h} \cdot \overline{\left(\frac{g \hat{h}_{z}+\hat{h} g_{z}}{\hat{h}^{2} g_{z}}\right)}\left(g+\overline{\left(\frac{\hat{h}_{z}}{g \hat{h}_{z}+\hat{h} g_{z}}\right)}\right)(z)
$$

for any $z \in \hat{U}$. We then have

$$
\hat{E}_{\bar{z}}=\hat{h} \overline{\left(\frac{\hat{h} g_{z} \hat{h}_{z z}-2 \hat{h}_{z}^{2} g_{z}-\hat{h} \hat{h}_{z} g_{z z}}{\hat{h}^{3} g_{z}^{2}}\right)}\left(1+|g|^{2}\right) .
$$

Thus $\left|\hat{E}_{\bar{z}}\right|=|f|\left(1+|g|^{2}\right)$, and therefore $\hat{E}_{\bar{z}}(z) \neq 0, \infty$ for every $z \in \hat{U}$. We deduce then with the aid of Theorem 2-5 that $\hat{E}$ generates a constant mean curvature one conformal immersion $\hat{X}: \hat{U} \rightarrow \boldsymbol{H}^{3}$ whose euclidean Gauss map is $\hat{E}$. We infer from Proposition 3-8 that the Weierstrass representation $(\hat{g}, \hat{f} d z)$ of the minimal immersion of $\hat{U}$ into $\boldsymbol{R}^{3}$ associated to $\hat{X}$ is given by

$$
\hat{g}=g, \quad \hat{f}=-\frac{\hat{h} g_{z} \hat{h}_{z z}-2 \hat{h}_{z}^{2} g_{z}-\hat{h} \hat{h}_{z} g_{z z}}{\hat{h}^{2} g_{z}^{2}} .
$$

Thus $\hat{g}=g$ and $\hat{f}=f$. Consequently, the immersions $X_{\mid \hat{U}}$ and $\hat{X}$ have the same metric and the same second fundamental form with respect their oriented euclidean Gauss map. This implies that $X_{\mid \hat{U}}$ and $\hat{X}$ differ by a positive isometry $J$ of $\boldsymbol{H}^{3}$, that is, $\hat{X}=J \circ X_{\mid \hat{U}}$. Thus $\hat{X}$ can be extended to the whole $U$ as a mean curvature one conformal immersion, since $X$ is defined on $U$. We call again $\hat{X}$ the conformal immersion defined on the whole $U$.

Let $\hat{G}$ be the hyperbolic Gauss map of $\hat{X}$. We infer from Proposition 3-6 that $\hat{G}_{z}=\hat{h}^{2} g_{z}$ on $\hat{U}$. Thus $\hat{h}^{2}=\hat{G}_{z} / g_{z}$ on $\hat{U}$. Since $\hat{G}$ and $g$ are defined on the whole $U$, we deduce again that $\hat{h}^{2}$ can be extended to a meromorphic map on $U$. Recall that $\hat{G}$ and $g$ have same order at any point of $U$ (see Corollary 2-9). This implies that the zeros and poles of $\hat{G}_{z} / g_{z}$ on $U$, if any, must have even multiplicity. As $U$ is simply connected, using the Monodromy Theorem, one can show that $\hat{G}_{z} / g_{z}$ has a well-defined square root meromorphic function on $U$. Thus $\hat{h}$ can be extended to a meromorphic function on $U$, which we call again $\hat{h}$. Then, by the analytic continuation, $\hat{h}$ satisfies Equation (3.15) on the whole $U$ as desired.

Finally, we show that $\hat{X}$ is generated by $\hat{E}$ on the whole $U$ and not only on $\hat{U}$. For this purpose we set

$$
U^{*}=\left\{z \in U ; \hat{h}(z) \neq 0, \infty, g(z) \neq \infty, g_{z}(z) \neq 0, \hat{h}_{z}(z) \neq 0\right\} .
$$


Observe that $U \backslash U^{*}$ consists of isolated points. Furthermore, note that $\hat{E}$ can be extended to a well-defined function on $U^{*}$. We have $\hat{E}(z) \neq \infty$ and $\hat{E}_{\bar{z}}(z) \neq 0$ for any $z \in U^{*}$, since $\left|\hat{E}_{\bar{z}}\right|=|f|\left(1+|g|^{2}\right)$. Now we set $\tilde{X}:=(\tilde{u}, \tilde{v}, \tilde{w}): U^{*} \rightarrow \boldsymbol{H}^{3}$, where

$$
(\tilde{u}+i \tilde{v})(z)=(\hat{G}-\tilde{w} \hat{E})(z), \quad \tilde{w}(z)=\frac{\left|\hat{h}^{2} g_{z}\right|^{2}}{\left|g \hat{h}_{z}+\hat{h} g_{z}\right|^{2}+\left|\hat{h}_{z}\right|^{2}}
$$

for every $z \in U^{*}$. A simple computation then shows that $\tilde{w}$ satisfies (2.1) in Remark 2-2 with $\hat{E}$ instead of $E$.

Since $\hat{E} \neq \infty$ on $U^{*}$, the proof of Theorem 2-5 shows that $\tilde{X}: U^{*} \rightarrow \boldsymbol{H}^{3}$ is a mean curvature one conformal immersion having the euclidean Gauss map $\hat{E}$ and the hyperbolic Gauss map $\hat{G}$. As $\hat{X}=\tilde{X}$ on $\hat{U}$, we conclude that $\hat{X}=\tilde{X}$ on $U^{*}$. Since $U \backslash U^{*}$ has only isolated points, we deduce with an argument of continuity that the euclidean Gauss map of $\hat{X}$ is $\hat{E}$ and $\hat{X}$ is defined by $\hat{E}$ on the whole $U$. That is, for every $z \in U$

$$
\hat{w}(z)=\frac{\left|\hat{h}^{2} g_{z}\right|^{2}}{\left|g \hat{h}_{z}+\hat{h} g_{z}\right|^{2}+\left|\hat{h}_{z}\right|^{2}}(z), \quad \hat{G}_{z}(z)=\hat{h}^{2} g_{z}(z),
$$

and

$$
\hat{E}(z)=\hat{h} \cdot \overline{\left(\frac{g \hat{h}_{z}+\hat{h} g_{z}}{\hat{h}^{2} g_{z}}\right)}\left(g+\overline{\left(\frac{\hat{h}_{z}}{g \hat{h}_{z}+\hat{h} g_{z}}\right)}\right)(z), \quad(\hat{u}+i \hat{v})(z)=(\hat{G}-\hat{w} \hat{E})(z) .
$$

This shows that the euclidean Gauss map of $X$ is given by (3.5).

Henceforth, (3.6), (3.7), (3.9), (3.10) and (3.11) follow from Proposition 3-6 on the subdomain $\tilde{U}=\{z \in U ; E(z) \neq \infty\}$. Since $U \backslash \tilde{U}$ consists of isolated points, we infer that these relations hold on the whole $U$. On the other hand, (3.8) follows from Proposition 1-2. Lastly, we deduce from Proposition 2-7 that $K=-|\tilde{\Phi}|^{2} /\left|E_{\bar{z}}\right|^{4}$ on $\tilde{U}$. Hence, using the expression of $E_{\bar{z}}$ (see the proof of Proposition 3-6), we easily find the desired formula for $K$ on $\tilde{U}$ and, by continuity, on the whole $U$. Relations (3.13) and (3.14) follow from Proposition 3-8. This completes the proof of the theorem.

REMARK 3-10. We note that it is possible to verify Small's formula using our representation (see $[\mathrm{S}]$ ). Indeed, under the notation of the present paper, $T=g$ is the secondary Gauss map and $h$ is equal to $\sqrt{d G / d g}$ (see Proposition 3-6). Then the meromorphic representation formula implies Small's formula for null meromorphic map $F: M \rightarrow \operatorname{PSL}(2 C)$ :

$$
F=\left(\begin{array}{cc}
G \frac{d a}{d G}-a & G \frac{d b}{d G}-b \\
\frac{d a}{d G} & \frac{d b}{d G}
\end{array}\right), \quad\left(a:=\sqrt{\frac{d G}{d g}}, \quad b:=-g \sqrt{\frac{d G}{d g}}\right) .
$$

Lemma 3-11. Let $U \subset \boldsymbol{C}$ be a simply connected domain and $X: U \rightarrow \boldsymbol{H}^{3}$ a nontotally umbilic conformal immersion. Assume that $X$ has mean curvature one with respect to E. Consider the meromorphic data $h$ and $T$ of $X$ given by Theorem 3-9. Let $z_{0} \in U$ be a point such that $E\left(z_{0}\right) \neq \infty$. Then $h$ is holomorphic at $z=z_{0}$. Moreover, $h$ vanishes at $z=z_{0}$ if and only if $T$ has a pole there whose multiplicity is the same as the order of $h$ at $z_{0}$. 
Conversely, let $z_{0} \in U$ be a point where $h$ is holomorphic and such that $h$ vanishes there if and only $T$ has a pole at $z=z_{0}$ whose multiplicity is equal to the order of $h$ at $z_{0}$. Then we have $E\left(z_{0}\right) \neq \infty$.

PROOF. In order to simplify the notation, let us set $z_{0}=0$. Consider the Weierstrass representation $(g, f d z)$ of the minimal immersion in $\boldsymbol{R}^{3}$ associated to the mean curvature one conformal immersion $X: U \rightarrow \boldsymbol{H}^{3}$. We have seen in Proposition 3-8 that we can choose $g=T$. We deduce with the aid of Corollary 2-9 that $T$ and $G$ have the same order at any point and, in particular at $z=0$. Recall that $G_{z}=h^{2} T_{z}$ (see relation (3.7) of Theorem 3-9). Since $G(0) \neq \infty$ (because $E(0) \neq \infty$ ) we infer that $G_{z}$ is holomorphic at $z=0$.

Assume that $h$ has a pole at $z=0$. Thus $h(z)=z^{p} H(z)$, where $-p \in N^{*}$ and $H$ is a holomorphic function at $z=0$ with $H(0) \neq 0$. As $G_{z}=h^{2} T_{z}$, we deduce that $T$ is holomorphic at $z=0$ and $T_{z}$ must vanish there. Thus $G_{z}$ must also vanish there with the same order as $T_{z}$. This is impossible, since $h$ is supposed to have a pole at $z=0$. Therefore $h$ is holomorphic at $z=0$.

Assume that $h$ vanishes at $z=0$. Since $G$ and $T$ have the same order at $z=0$, we deduce that $T$ cannot be holomorphic at this point. Therefore, $T$ must have a pole at $z=0$. Thus, $T(z)=z^{q} t(z)$ and $h(z)=z^{p} H(z)$, where $p,-q \in N^{*}$ and $t$ and $H$ are holomorphic functions with $t(0) \neq 0$ and $H(0) \neq 0$. Since $G_{z}$ is holomorphic at $z=0$, and $G$ and $T$ have the same order at this point, we must have $2 p+q-1 \geq 0$ and $2 p+q=-q$. That is, $q=-p$, which shows that $T$ must have a pole at $z=0$ whose multiplicity is equal to the order of $h$ there.

Finally, assume that $T$ has a pole at $z=0$. Thus we have $T(z)=z^{q} t(z)$, where $-q \in N^{*}$ and $t$ is a holomorphic function with $t(0) \neq 0$. Then the previous argument shows that $h$ must vanish at $z=0$ and the order of $h$ there must be equal to the order of $T$ at $z=0$. This completes the proof of the first statement of the lemma.

Conversely, let $z_{0} \in U$ be any point, and suppose that $h$ is holomorphic at $z=z_{0}$. If $T$ is also holomorphic at $z_{0}$, we deduce from the relation $G_{z}=h^{2} T_{z}$ that $G$ is also holomorphic there. Therefore, in this case we have $E\left(z_{0}\right) \neq \infty$. Assume now that $T$ has a pole at $z=z_{0}$ with multiplicity $n \in N^{*}$ and that $h$ vanishes there with multiplicity $n$. We infer from the relation $G_{z}=h^{2} T_{z}$ that $G$ is holomorphic at $z_{0}$. Consequently, we must have $E\left(z_{0}\right) \neq \infty$. This achieves the proof.

Recall that Equation (*) is a global equation (see Remark 2-2-(3)). Now, consider a Riemann surface $S$ and let $h$ and $T$ be two meromorphic functions on $S$ such that $h \neq 1 /(\alpha T+$ $\beta$ ) for any complex numbers $\alpha, \beta$. Then $w:=\left|h^{2} T_{z}\right|^{2} /\left(\left|T h_{z}+h T_{z}\right|^{2}+\left|h_{z}\right|^{2}\right)$ defines a global function on $S$ with isolated zeros and "poles". Thus Theorem 3-9 leads to a global representation:

THEOREM 3-12 (Representation Theorem). Let $S$ be a Riemann surface and let $h$ and $T$ be non constant meromorphic functions on $S$ such that $h \neq 1 /(\alpha T+\beta)$ for any complex 
numbers $\alpha, \beta$. Set

$$
w(z)=\frac{\left|h^{2} T_{z}\right|^{2}}{\left|T h_{z}+h T_{z}\right|^{2}+\left|h_{z}\right|^{2}}, \quad E=h \cdot \overline{\left(\frac{T h_{z}+h T_{z}}{h^{2} T_{z}}\right)}\left(T+\overline{\left(\frac{h_{z}}{T h_{z}+h T_{z}}\right)}\right)
$$

and $S^{*}=\left\{z \in S ;\left|E_{\bar{z}}\right| \neq 0, \infty\right\}$. Assume that

$$
\int_{\gamma} h^{2} T_{z} d z=0
$$

for every closed path $\gamma \subset S$ on which neither $h$ nor $T$ have poles, that is, the 1 -form $h^{2} T_{z} d z$ has a global primitive $G$ on $S$. Set

$$
(u+i v)(z):=(G-w E)(z), \quad z \in S^{*} .
$$

Then the function $X:=(u, v, w): S^{*} \rightarrow \boldsymbol{H}^{3}$ defines a mean curvature one conformal immersion whose euclidean Gauss map is $E$ and the hyperbolic Gauss map is $G$ (recall that $S \backslash S^{*}$ is a discrete set). Furthermore, the geometric quantities of $X$ are given by (3.9) through (3.14) of Theorem 3-9.

Observe that when $S$ is simply connected, (3.17) means that the function $h^{2} T_{z}$ does not have non-zero residues.

PROOF. It follows from (3.17) that there exists a meromorphic function $G$ on $U$ such that $d G=h^{2} d T$, that is, locally, $G_{z}=h^{2} T_{z}$. Clearly, the set of zeros and "poles" of $w$ is contained in the set of zeros and poles of $h, d h, T$ and $d T$. Therefore the zeros and "poles" of $w$ are isolated points. We set

$$
S^{+}:=\left\{z \in S ;\left|E_{\bar{z}}(z)\right| \neq 0, \infty, E(z) \neq \infty, w(z) \neq 0, \infty\right\} .
$$

A straightforward verification then shows that

$$
w_{\bar{z}}=-w \frac{\bar{E} E_{\bar{z}}}{1+E \bar{E}}
$$

on $S^{+}$. Hence it can be inferred from the proof of Theorem 2-5 that $X$ is a mean curvature one conformal immersion of $S^{+}$into $\boldsymbol{H}^{3}$ whose euclidean (resp. hyperbolic) Gauss map is $E$ (resp. $G$ ). Let $z_{0} \in S^{*}$, and set $g=T$ and

$$
f=-\frac{1}{T_{z}} \cdot \frac{h T_{z} h_{z z}-2 h_{z}^{2} T_{z}-h h_{z} T_{z z}}{h^{2} T_{z}} .
$$

We then get $|f|\left(1+|g|^{2}\right)=\left|E_{\bar{z}}\right|$. Thus $(g, f d z)$ define a mean curvature one conformal immersion $X_{0}$ of a simply connected neighborhood of $z_{0}$, say $U_{0}$, into $\boldsymbol{H}^{3}$. Note that when restricted to the set $S^{+} \cap U_{0}, X_{0}$ and $X$ differ by a positive isometry of $\boldsymbol{H}^{3}$. Henceforth, $X$ can be extended to any point $z_{0} \in S^{*}$. This shows that for any $z_{0} \in S^{*}$, we must have $w\left(z_{0}\right) \neq 0, \infty$. This achieves the proof of the theorem. 
THEOREM 3-13. Let $U \subset C$ be a simply connected domain and $E: U \rightarrow C$ a $C^{2}$ map, which is neither holomorphic nor anti-holomorphic and satisfies

$$
E_{z \bar{z}}=\frac{\bar{E}}{1+E \bar{E}} E_{z} E_{\bar{z}} .
$$

Then there exist a holomorphic function $h$ and a meromorphic function $T$ on $U$ such that

$$
E=h \cdot \overline{\left(\frac{T h_{z}+h T_{z}}{h^{2} T_{z}}\right)}\left(T+\overline{\left(\frac{h_{z}}{T h_{z}+h T_{z}}\right)}\right) .
$$

Moreover, for any point $z_{0} \in U, T$ has a pole there if and only if $h$ vanishes at $z_{0}$, and $T$ and $h$ have the same order at this point.

Proof. Assume first that $E_{\bar{z}}(z) \neq 0$ for every $z \in U$. Then it follows from Theorem 2-5 that $E$ generates a mean curvature one conformal immersion from $U$ into $\boldsymbol{H}^{3}$ whose euclidean Gauss map is $E$. Then we apply Theorem 3-9 to conclude that there exists meromorphic functions $h$ and $T$ on $U$ satisfying the conditions.

Assume now that $E_{\bar{z}}$ vanishes at some points. Since $E$ is not holomorphic, the points where $E_{\bar{z}}$ vanishes are isolated points (see Lemma 2-3). Set $U^{*}=\left\{z \in U ; E_{\bar{z}}(z) \neq 0\right\}$. Note that Theorem 2-5 allows us to construct a $C^{2}$ map $X: U \rightarrow \boldsymbol{H}^{3}$ such that its restriction to $U^{*}$ is a mean curvature one conformal immersion whose euclidean Gauss map is $E$. Consequently, each zero of $E_{\bar{z}}$ is a branch point (that is, a singularity) of $X$. Since $E$ is a $C^{2}$ map, each branch point of $X$ has a well-defined unit normal vector.

Observe that, beginning on a simply connected domain of $U^{*}$ and using the analytic continuation principle, we can construct a (possibly multi-valued) map $Y: U^{*} \rightarrow \boldsymbol{R}^{3}$ which is a minimal conformal (possibly multi-valued) immersion, locally associated to $X$. Let $(g, f d z)$ be the Weierstrass representation of $Y$. Thus $g$ and $f$ are (possibly multi-valued) meromorphic functions on $U^{*}$.

We claim that $Y$ can be extended to a branched minimal immersion from $U$ into $\boldsymbol{R}^{3}$, branched at each zero point of $E_{\bar{z}}$. Let $z_{0} \in U$ be a branch point of $X$, that is, a zero point of $E_{\bar{z}}$. Let $D \subset U$ be a small round disk centered at $z_{0}$ and such that $z_{0}$ is the only branch point of $X$ contained in $D$. We denote again by $Y$ the restriction of the (possibly multi-valued) minimal immersion $Y$ on $D^{*}=D \backslash\left\{z_{0}\right\}$. Then define the new (possibly multi-valued) map $\tilde{Y}: D^{*} \rightarrow \boldsymbol{R}^{3}$ by setting $\tilde{Y}(z)=\lim _{\theta \rightarrow 2 \pi, \theta<2 \pi} Y\left(z_{0}+e^{i \theta}\left(z-z_{0}\right)\right)$ for any $z \in D^{*}$. Note that the (possibly multi-valued) maps $Y$ and $\tilde{Y}$ are locally associated to $X$ (restricted to $D^{*}$ ). Therefore they share the same metric and the same second fundamental form. We deduce that $Y$ and $\tilde{Y}$ differ by a positive isometry $I$ of $\boldsymbol{R}^{3}$. Thus, $I$ is a translation composed by a rotation $R$ around a straight line of $\boldsymbol{R}^{3}$. Up to a positive isometry of $\boldsymbol{R}^{3}$, we can assume that $R$ is a rotation around the vertical $x_{3}$-axis. Thus there exists a real number $\alpha$ with $0 \leq \alpha<1$ such that $\lim _{\theta \rightarrow 2 \pi, \theta<2 \pi} g\left(z_{0}+e^{i \theta}\left(z-z_{0}\right)\right)=e^{2 \pi i \alpha} g(z)$ for every $z \in D^{*}$. Hence $g(z) \cdot\left(z-z_{0}\right)^{-\alpha}$ is a well-defined meromorphic function on $D^{*}$. We want to show that $\alpha=0$.

Since $E$ is $C^{2}$ at $z=z_{0}$, we infer that $E$ and $E_{z}$ both have finite limits when $z$ goes to $z_{0}$. Hence we deduce with the aid of Proposition 2-7 that the expression $K(z) d s^{2}=$ 
$\left(-4\left|E_{z}\right|^{2} /(1+E \bar{E})^{2}\right)|d z|^{2}$ is bounded near $z=z_{0}$. Therefore, $X$ has finite total curvature in a neighborhood of $z=z_{0}$. Now, it follows from the proof of Proposition 4 in [B] that $g(z) \cdot\left(z-z_{0}\right)^{-\alpha}$ extends continuously to $z=z_{0}$. Suppose that $\alpha \neq 0$. Then $g(z)$ has a limit when $z$ goes to $z_{0}$, which is 0 or $\infty$. Up to a positive isometry of $\boldsymbol{R}^{3}$, we can choose this limit to be equal to 0 . Thus there exist an integer $p \in N$ and a meromorphic function $\psi$ on $D$ such that $g(z)=\left(z-z_{0}\right)^{\alpha+p} \psi(z)$ with $\psi\left(z_{0}\right) \in C \backslash\{0\}$. As $E$ is a $C^{2}$ map, we deduce with the aid of Proposition 2-7 that the Hopf function $\tilde{\Phi}$ is well-defined on $D$. Since $\tilde{\Phi}=-2 f g_{z}$ (see Corollary 2-9), we infer that the restriction of $f$ on $D^{*}$ must have the following form: $f(z)=\left(z-z_{0}\right)^{-\alpha} \varphi(z)$, where $\varphi$ is a holomorphic function on $D$. Let us set $T(z)=g(z)$. As in the proof of Theorem 3-9, we can find a meromorphic function $h$ on a simply connected domain $\tilde{D}$ of $D^{*}$ satisfying the ordinary differential equation

$$
f=-\frac{1}{g_{z}} \cdot \frac{h g_{z} h_{z z}-2 h_{z}^{2} g_{z}-h h_{z} g_{z z}}{h^{2} g_{z}} .
$$

Therefore, the data $(h, T)$ define a mean curvature one conformal immersion $\tilde{X}: \tilde{D} \rightarrow \boldsymbol{H}^{3}$ (see Theorem 3-12). Since $\tilde{X}$ and $X$ have the same metric and the same second fundamental form, we deduce that they differ by a positive isometry of $\boldsymbol{H}^{3}$, and hence $\tilde{X}$ is globally defined on $D$ (branched at $z=z_{0}$ ). Finally, since the hyperbolic Gauss map $\tilde{G}$ of $\tilde{X}$ is well-defined on $D$ and $\tilde{G}_{z}=h^{2} g_{z}$ on $\tilde{D}$, we infer that $h$ can be extended to a meromorphic multi-valued function on $D^{*}$, with $h^{2}$ having the following form: $h^{2}(z)=\left(z-z_{0}\right)^{q-\alpha} H(z)$, where $q \in \boldsymbol{Z}$ and $H$ is a meromorphic function on $D$ with $H\left(z_{0}\right) \neq 0, \infty$. Note that the metric $d s^{2}$ must have a zero at $z=z_{0}$. On the other hand, Theorem 3-9 shows that

$$
d s^{2}=\frac{\left|h g_{z} h_{z z}-2 h_{z}^{2} g_{z}-h h_{z} g_{z z}\right|^{2}}{\left|h g_{z}\right|^{4}}\left(1+|g|^{2}\right)^{2}|d z|^{2} .
$$

Thus the expression $A(z)=\left(h g_{z} h_{z z}-2 h_{z}^{2} g_{z}-h h_{z} g_{z z} /\left(h g_{z}\right)^{2}\right)(z)$ must vanish at $z=z_{0}$. Observe that

$$
A=\left(\left(\frac{h_{z}}{h}\right)_{z}-\left(\frac{h_{z}}{h}\right)^{2}\right) \cdot \frac{1}{g_{z}}+\left(\frac{h_{z}}{h}\right) \cdot\left(\frac{1}{g_{z}}\right)_{z} .
$$

Recall that $h^{2}(z)=\left(z-z_{0}\right)^{q-\alpha} H(z)$ and $g(z)=\left(z-z_{0}\right)^{p+\alpha} \psi(z)$ with $q \in Z, p \in N$, $0<\alpha<1$, where $H$ and $\psi$ are meromorphic on $D$ with $H\left(z_{0}\right), \psi\left(z_{0}\right) \neq 0, \infty$. We deduce that

$$
A(z)=-\frac{(q-\alpha)(q+2 p+\alpha)}{4(p+\alpha) \psi\left(z_{0}\right)} \cdot \frac{1}{\left(z-z_{0}\right)^{p+\alpha+1}}+o\left(\frac{1}{\left(z-z_{0}\right)^{p+\alpha}}\right) .
$$

Therefore, as $0<\alpha<1, A(z)$ cannot vanish at $z=z_{0}$. We deduce that $\alpha=0$, that is, $f$ and $g$ are globally defined on $D$. Furthermore, since the metric is singular at $z=z_{0}$, the function $f$ must vanish at $z=z_{0}$. Thus the map $Y$ extend to a well-defined minimal conformal immersion $Y: D \rightarrow \boldsymbol{R}^{3}$ branched at $z=z_{0}$ as claimed.

Now, we are going to show that $g$ and $G$ have the same order at $z=z_{0}$. Observe that $G\left(z_{0}\right) \neq \infty$, since $E\left(z_{0}\right) \neq \infty$. Recall that $f g_{z}=-E_{z} \overline{\left(E_{\bar{z}}\right)} /(1+E \bar{E}$ ) (see Corollary 2-9). Also, we have the relation $|f|\left(1+|g|^{2}\right)=\left|E_{\bar{z}}\right|$ proved in Corollary 2-9. Hence the function 
$\left|f / E_{\bar{z}}\right|$ can be continuously extended to $z=z_{0}$ by setting $\left|f / E_{\bar{z}}\right|\left(z_{0}\right)=1\left(\right.$ since $\left.g\left(z_{0}\right)=0\right)$. Therefore, we have

$$
f g_{z}=-\frac{E_{z} \overline{\left(E_{\bar{z}}\right)}}{1+E \bar{E}}=-\frac{G_{z} \overline{\left(E_{\bar{z}}\right)}}{w(1+E \bar{E})} .
$$

Hence, $\left|G_{z} / g_{z}\right|=w(1+E \bar{E}) \cdot\left|f / E_{\bar{z}}\right|$. We conclude that if $g_{z}$ or $G_{z}$ vanishes at $z=z_{0}$, then they both vanish with the same order.

Clearly, the discussion above holds for every branch point of $X$. Thus, $Y: U \rightarrow R^{3}$ is a well-defined branched minimal immersion with the same branch points as that of $X$. Thus the Weierstrass representation $(g, f d z)$ is well-defined on the whole $U$, and $g$ and $G$ have the same order at any point of $U$ (see Corollary 2-9 for the regular points of $X$, and see the argument above for the branched points of $X$ ). Now the proof of assertion (3.5) of Theorem 3-9 ensures that there exist meromorphic functions $h$ and $T$ on $U$ such that

$$
E=h \cdot \overline{\left(\frac{T h_{z}+h T_{z}}{h^{2} T_{z}}\right)}\left(T+\overline{\left(\frac{h_{z}}{T h_{z}+h T_{z}}\right)}\right) .
$$

The last statement of the theorem as well as the fact that $h$ is holomorphic on $U$ follows from Lemma 3-11, since $E \neq \infty$ on the whole $U$ by assumption. This completes the proof of the theorem.

REMARK 3-14. Assume that $X: U \rightarrow \boldsymbol{H}^{3}$ has mean curvature one and let $(g, f d z)$ be the Weierstrass representation of the associated minimal immersion in $\boldsymbol{R}^{3}$. For any meromorphic function $W$, let us denote by $S(W)$ the Schwarzian derivative of $W$, that is,

$$
S(W)=\frac{d}{d z}\left(\frac{W_{z z}}{W_{z}}\right)-\frac{1}{2}\left(\frac{W_{z z}}{W_{z}}\right)^{2} .
$$

Then we can easily verify the following relation

$$
S(G)=S(g)+\tilde{\Phi}=S(g)-2 f g_{z},
$$

obtained by Umehara and Yamada (see [U-Y, 1], (2.6)).

Indeed, we infer from Theorem 3-9 that

$$
S(G)=2 \frac{h_{z z}}{h}-4 \frac{h_{z}^{2}}{h^{2}}-2 \frac{h_{z}}{h} \cdot \frac{T_{z z}}{T_{z}}+\frac{T_{z z z}}{T_{z}}-\frac{3}{2} \frac{T_{z z}^{2}}{T_{z}^{2}}, \quad S(g)=\frac{T_{z z z}}{T_{z}}-\frac{3}{2} \frac{T_{z z z}^{2}}{T_{z}^{2}} .
$$

Therefore, we have

$$
S(g)-2 f g_{z}=\frac{T_{z z z}}{T_{z}}-\frac{3}{2} \frac{T_{z z}^{2}}{T_{z}^{2}}+2 \frac{h_{z z}}{h}-4 \frac{h_{z}^{2}}{h^{2}}-2 \frac{h_{z}}{h} \cdot \frac{T_{z z}}{T_{z}}=S(G) .
$$

EXAMPLE 3-15. We have seen in Example 2-13 that the euclidean Gauss map $E$ of the Enneper cousins is given by

$$
E(z)=\frac{\gamma}{\bar{\gamma}} z e^{-\gamma z} e^{\overline{\gamma z}}\left(\frac{\bar{\gamma}}{z}-1+\overline{\gamma z}\right)
$$


for any non-zero complex number $\gamma$. Conversely, consider the mean curvature one conformal immersion generated by $E$ (see Theorem 2-5). As a verification, we are looking for the meromorphic data $(h, T)$ stated in Theorem 3-9, and then see that the Weierstrass representation of the associated minimal immersion is, up to a positive isometry of $\boldsymbol{H}^{3}, g(z)=z$ and $f(z) \equiv \alpha$, where $\alpha=\gamma^{2}$. This will show that the mean curvature one conformal immersions generated by $E$ are the Enneper cousins.

We first call $\hat{h}(z)=\gamma z e^{-\gamma z} / \bar{\gamma}$ and $\hat{T}(z)=-1+\bar{\gamma} / z$ for any $z \in C$. Let $a$ and $b$ be any complex numbers with $a \neq 0$ and consider the meromorphic functions $R$ and $S$ given in Proposition 3-5. Set $\hat{E}=\hat{h} \bar{S}(\hat{T}+\bar{R})$. Then a simple computation shows that $\hat{E}=E$ if and only if $a=b=1 / \bar{\gamma}$. Now, we set $h(z)=\hat{h}(z) / a=\gamma z e^{-\gamma z}$ and $T(z)=b+a \hat{T}(z)=1 / z$. Then a straightforward calculation shows that

$$
h \cdot \overline{\left(\frac{T h_{z}+h T_{z}}{h^{2} T_{z}}\right)}\left(T+\overline{\left(\frac{h_{z}}{T h_{z}+h T_{z}}\right)}\right)=E .
$$

Then we easily infer that

$$
-\frac{1}{T_{z}} \cdot \frac{h T_{z} h_{z z}-2 h_{z}^{2} T_{z}-h h_{z} T_{z z}}{h^{2} T_{z}}(z)=-\gamma^{2} z^{2}
$$

We deduce with (3.13) and (3.14) of Theorem 3-9 that the Weierstrass representation of the associated minimal immersion in $\boldsymbol{R}^{3}$ is $\tilde{g}(z)=1 / z$ and $\tilde{f}(z) d z=-\gamma^{2} z^{2} d z$. Finally, let $I$ be the euclidean reflection in $\boldsymbol{R}^{3}$ with respect to the $x_{1}$-axis (where $\left(x_{1}, x_{2}, x_{3}\right)$ are the coordinates of $\boldsymbol{R}^{3}$ ). Let $\tilde{Y}: \boldsymbol{C} \rightarrow \boldsymbol{R}^{3}$ be the conformal minimal immersion generated by $(\tilde{g}, \tilde{f} d z)$. We call $(g, f d z)$ the Weierstrass representation of the conformal minimal immersion $Y=I \circ \tilde{Y}$. Hence a straightforward computation shows that $g(z)=z$ and $f(z)=\alpha$, where $\alpha=\gamma^{2}$, and we recognize the Weierstrass representation of the Enneper surfaces.

4. Examples. Let us set $U=C$ and choose the holomorphic data $h(z)=e^{\gamma z}$ and $T(z)=b+e^{z}$, where $b, \gamma \in C$. Define $E$ as in (3.18) in Theorem 3-13, that is,

$$
E(z)=e^{\gamma z} e^{-\overline{\gamma z}} \cdot\left(\overline{b \gamma e^{-z}+(1+\gamma)}\right) \cdot\left(b+e^{z}+\overline{\left(\frac{\gamma}{b \gamma+(1+\gamma) e^{z}}\right)}\right) .
$$

A straightforward computation then leads to $E_{\bar{z}}(z)=-\overline{\gamma(1+\gamma)} e^{\gamma z-\overline{(\gamma+1) z}}\left(1+\left|b+e^{z}\right|^{2}\right)$ and $\left(h T_{z} h_{z z}-2 h_{z}^{2} T_{z}-h h_{z} T_{z z}\right)(z)=-\gamma(\gamma+1) e^{z+2 \gamma z}$.

Combining with Theorem 3-9, we deduce that for any $\gamma \in C \backslash\{-1,0\}$, the data $(h, T)$ give rise to a mean curvature one conformal immersion $X: \boldsymbol{C} \rightarrow \boldsymbol{H}^{3}$ whose metric is

$$
d s^{2}=\left|E_{\bar{z}}(z) d z\right|^{2}=\left|\gamma(1+\gamma) e^{-z}\right|^{2}\left(1+\left|b+e^{z}\right|^{2}\right)^{2}|d z|^{2} .
$$

We infer easily that $X$ is a complete immersion for any complex numbers $b$ and $\gamma$ with $\gamma \neq-1,0$. Let us call $(g, f d z)$ the Weierstrass representation of the associated minimal immersion $Y: \boldsymbol{C} \rightarrow \boldsymbol{R}^{3}$. We infer from Theorem 3-9 that $g(z)=T(z)=b+e^{z}$ and $f(z)=\gamma(\gamma+1) e^{-z}$. When $b=0$, we recognize the catenoid-helicoid family. We deduce 
that, up to a positive isometry of $\boldsymbol{H}^{3}$, if $b=0$, then $X$ is one of the immersions seen in Example 2-12.

From now on, we assume $b \neq 0$. From Theorem 3-9 we get

$$
\begin{aligned}
& w(z)=e^{\gamma z+\overline{\gamma z}} \cdot \frac{\left|e^{z}\right|^{2}}{\left|b \gamma+(1+\gamma) e^{z}\right|^{2}+|\gamma|^{2}}, \quad G_{z}(z)=e^{(2 \gamma+1) z}, \\
& (u+i v)(z)=(G-w E)(z) .
\end{aligned}
$$

First case: $\gamma=-1 / 2$. In this case, $G_{z} \equiv 1$. Thus we can choose $G(z)=z$. Furthermore, by setting $\gamma=-1 / 2$ in the formulas above, we get

$$
\left\{\begin{array}{l}
\left.E(z)=\frac{e^{-z / 2}}{2} \overline{\left(-b e^{-z / 2}+e^{z / 2}\right.}\right)\left(b+e^{z}+\overline{\left(\frac{1}{b-e^{z}}\right)}\right), \\
w(z)=4 \frac{\left|e^{z}\right|}{1+\left|b-e^{z}\right|^{2}}, \\
(u+i v)(z)=z+\frac{2}{1+\left|b-e^{z}\right|^{2}}\left(\left(b+e^{z}\right)\left(\overline{b-e^{z}}\right)+1\right) .
\end{array}\right.
$$

It follows that for any $z \in C, w(z+2 \pi i)=w(z), E(z+2 \pi i)=E(z), G(z+2 \pi i)=$ $G(z)+2 \pi i$ and $(u+i v)(z+2 \pi i)=(u+i v)(z)+2 \pi i$. Thus

$$
X(z+2 \pi i)=X(z)+(0,2 \pi, 0) .
$$

This shows that the surface $X(C)$ is invariant under the horizontal translations $(u+i v, w) \rightarrow$ $(u+i v, w)+(2 \pi i, 0)$ of $\boldsymbol{H}^{3}$. Therefore, $X(\boldsymbol{C})$ is invariant under a discrete subgroup of parabolic isometries of $\boldsymbol{H}^{3}$ isometric to $\boldsymbol{Z}$.

In Figure 1-a, we draw a piece of a fundamental domain of the surface corresponding to $b=1 / 2$. We draw also three fundamental domains of the same surface in Figure 1-b. Observe that for each $b \in C$ we get a dual of the Enneper cousins.

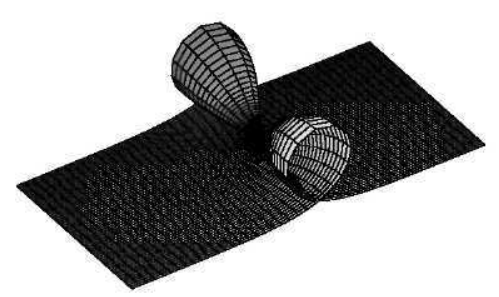

FIGURE 1-a.

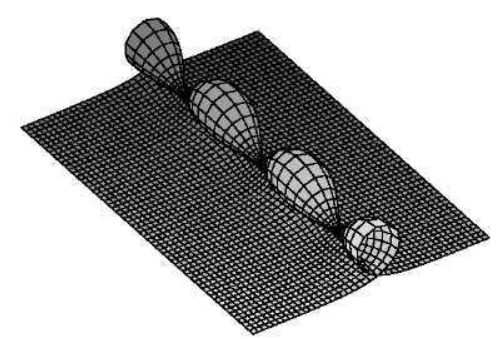

FIGURE 1-b

From now on, we assume $\gamma \neq-1 / 2$. Hence we can choose $G(z)=e^{(2 \gamma+1) z} /(2 \gamma+1)$. Thus

$$
(u+i v)(z)=e^{(2 \gamma+1) z}\left(\frac{1}{2 \gamma+1}-\frac{\left(b+e^{z}\right)\left(\overline{b \gamma+(1+\gamma) e^{z}}\right)+\bar{\gamma}}{\left|b \gamma+(1+\gamma) e^{z}\right|^{2}+|\gamma|^{2}}\right) .
$$


Therefore, for any $z \in C$ we have

$$
(u+i v)(z+2 \pi i)=e^{4 \pi i \gamma}(u+i v)(z), \quad w(z+2 \pi i)=e^{-4 \pi \operatorname{Im} \gamma} \cdot w(z) .
$$

For any real number $\lambda$, let $R_{\lambda}$ be the rotation around the $w$-axis whose argument is $\lambda$ and let $H_{\lambda}$ be the homothety with respect to 0 and ratio $e^{\lambda}$. Note that $R_{\lambda}$ (resp. $H_{\lambda}$ ) is an elliptic (resp. hyperbolic) isometry of $\boldsymbol{H}^{3}$.

Second case: $\operatorname{Re}(\gamma)=0$. Then $X(z+2 \pi i)=H_{-4 \pi} \operatorname{Im}(\gamma)(X(z))$. That is, $X$ is invariant under a discrete subgroup of hyperbolic isometry of $\boldsymbol{H}^{3}$ isometric to $\boldsymbol{Z}$.

Third case: $\gamma \in \boldsymbol{R} \backslash\{-1,0,-1 / 2\}$. In this case we have $X(z+2 \pi i)=R_{4 \pi \gamma}(X(z))$. That is, $X$ is invariant under a subgroup of elliptic isometries of $\boldsymbol{H}^{3}$. Recall that when $b=0$, $X$ is invariant under a reflection in $\boldsymbol{H}^{3}$ for any real $\gamma$ such that $\gamma(\gamma+1)>-1 / 4$, that is, $X$ parametrizes the catenoids cousin (see Example 2-12). This suggests to look for symmetries when $b \neq 0$, for any $\gamma \in \boldsymbol{R} \backslash\{-1,0,-1 / 2\}$. Let $R>0$ be a positive real number and let $S$ be the reflection in $\boldsymbol{H}^{3}$ with respect to the geodesic plane $\left\{(u, v, w) \in \boldsymbol{H}^{3} ; u^{2}+v^{2}+w^{2}=\right.$ $R^{2}$ ). The reflection $S$ is a negative isometry (that is, a orientation reverting isometry) of $\boldsymbol{H}^{3}$. Observe that if $S(X(C))=X(C)$, then there exists a orientation reverting conformal transformation of $\boldsymbol{C}$, say $\varphi(z)$, such that $S \circ X=X \circ \varphi$. Thinking of the catenoids cousin, we look for $\varphi$ with the following form: $\varphi(z)=-\bar{z}+a$, where $a \in \boldsymbol{R}$. Then a straightforward computation shows that if $a=\log \left(1+|b|^{2}\right)$, then $\varphi^{*}(d s)=d s$. Moreover, the relation $S \circ X=X \circ \varphi$ leads to $S_{\infty} \circ G=G \circ \varphi$, where $S_{\infty}$ is the restriction of $S$ to $\partial_{\infty} \boldsymbol{H}^{3}$. Thus we should have

$$
\overline{\left(\frac{R^{2}}{G(z)}\right)}=G(\varphi(z))=G(-\bar{z}+a)
$$

for any $z \in C$. Again a simple calculation shows that this condition is satisfied if and only if $R^{2}=e^{a(2 \gamma+1)} /(2 \gamma+1)^{2}$. Therefore, we take $a \in R$ such that $e^{a}=1+|b|^{2}$ and choose $R$ as above. Let us define the new immersion $\hat{X}=S \circ X \circ \varphi$. Clearly, $\hat{X}$ is a mean curvature one conformal immersion (with respect to the oriented euclidean Gauss map) sharing the same metric with $X$. Since the second fondamental form of $X$ is $\tilde{\Pi}=-\operatorname{Re}\left(\gamma(\gamma+1)(d z)^{2}\right)+d s^{2}$, we deduce that the second fondamental form of $\hat{X}$ is also $\tilde{\Pi}$. Thus $X$ and $\hat{X}$ coincide up to a positive isometry of $\boldsymbol{H}^{3}$. Nevertheless, since $S_{\infty} \circ G \circ \varphi=G$, we deduce that $X$ and $\hat{X}$ share the same hyperbolic Gauss map. Finally, we conclude $\hat{X}=X$, that is, $X$ is symmetric with respect to the geodesic plane $\left\{(u, v, w) \in \boldsymbol{H}^{3} ; u^{2}+v^{2}+w^{2}=R^{2}\right\}$.

(i) Assume $\gamma \in \boldsymbol{R} \backslash \boldsymbol{Q}$. Since $\gamma$ is irrational, $X$ is invariant under a subgroup of elliptic isometries of $\boldsymbol{H}^{3}$ isometric to $\boldsymbol{Z}$.

(ii) Assume $\operatorname{Im}(\gamma)=0$ and $\gamma \in Q \backslash\{0,-1,-1 / 2\}$. We have again $X(z+2 \pi i)=$ $R_{4 \pi \gamma}(X(z))$. Let us set $2 \gamma=p / q$, where $p \in Z^{*}, q \in N^{*}, p+2 q \neq 0$ (since $\gamma \neq-1$ ), $p+q \neq 0$ (since $\gamma \neq-1 / 2)$ and $p$ and $q$ without common factors. Therefore, $X(z+q 2 \pi i)=$ $X(z)$. Now we make the change of parameter $\zeta=e^{z / q}$, and let $\tilde{X}: C^{*} \rightarrow \boldsymbol{H}^{3}$ be the mean curvature one conformal immersion defined by $\tilde{X}(\zeta)=(\tilde{u}, \tilde{v}, \tilde{w})(\zeta):=X(z)$. A 
straighforward computation then leads to

$$
\left\{\begin{array}{l}
(\tilde{u}+i \tilde{v})(\zeta)=\zeta^{p+q}\left[\frac{q}{p+q}-2 q \cdot \frac{\left(b+\zeta^{q}\right)\left(\overline{b p+(2 q+p) \zeta^{q}}\right)+p}{\left|b p+(2 q+p) \zeta^{q}\right|^{2}+p^{2}}\right] \\
\tilde{w}(\zeta)=4 q^{2} \frac{|\zeta|^{p+2 q}}{\left|b p+(2 q+p) \zeta^{q}\right|^{2}+p^{2}} .
\end{array}\right.
$$

Let $U$ denote the function in the bracket in the formula of $(\tilde{u}+i \tilde{v})(\zeta)$ above, that is, $(\tilde{u}+$ $i \tilde{v})(\zeta)=\zeta^{p+q} \cdot U(\zeta)$. It is easily seen that $U(0)=0$ if and only if $p+2 q=0$. Also, $\lim _{\zeta \rightarrow \infty} U(\zeta)=-p q /(p+q)(p+2 q)$. Thus we infer from our assumption about $p$ and $q$ that $U(0) \neq 0$ and $\lim _{\zeta \rightarrow \infty} U(\zeta) \neq 0$. Furthermore, the Weierstrass representation of the minimal immersion in $\boldsymbol{R}^{3}$ locally associated to $\tilde{X}$ is $\tilde{g}(\zeta)=b+\zeta^{q}$ and $\tilde{f}(\zeta) d \zeta=(p(p+$ $\left.2 q) /\left(4 q \zeta^{q+1}\right)\right) d \zeta$. Observe that the Weierstrass data $(\tilde{g}, \tilde{f} d \zeta)$ define a conformal minimal immersion into $\boldsymbol{R}^{3}$ if and only if $b=0$. When $b=0$, we get the catenoids. Nevertheless, when $b \neq 0$, the data $(\tilde{g}, \tilde{f} d \zeta)$ define a singly periodic minimal immersion into $\boldsymbol{R}^{3}$. More precisely, $(\tilde{g}, \tilde{f} d \zeta)$ define a minimal immersion of $\boldsymbol{C}^{*}$ into the quotient space $\boldsymbol{R}^{3} / \boldsymbol{Z}$ whose total curvature is $-4 \pi q$. We deduce that $\tilde{X}$ has total curvature equals to $-4 \pi q$. Furthermore, as $\tilde{G}(\zeta)=(q / p+q) \zeta^{p+q}$, we infer that each end $(0$ and $\infty)$ is a regular end. From the expression of $(\tilde{u}+i \tilde{v})(\zeta)$ and the observation about the function $U(\zeta)$, we deduce that each end turns $|p+q|$ times around the $w$-axis. That is, the restriction of the orthogonal projection on the $(u, v)$-plane to each end is a $|p+q|$ covering map. Recall that theses surfaces are symmetric with respect to some geodesic plane (see the discussion above in the beginning of the third case). Then, it is easy to see that this geodesic plane is $\{(u, v, w) \in$ $\boldsymbol{H}^{3} ; u^{2}+v^{2}+w^{2}=R^{2}$ \} with $R^{2}=t^{p+q} \cdot q^{2} /(p+q)^{2}$, where $t$ is the positive real number such that $t^{q}=1+|b|^{2}$. Namely, we have $\left(S_{R} \circ \tilde{X}\right)(\zeta)=\tilde{X}(t / \bar{\zeta})$ for every $\zeta \in C^{*}$, where $S_{R}$ is the reflection about the half-sphere in $\boldsymbol{H}^{3}$ centered at 0 and radius $R$. In Figure 2 we draw the half part of the surface with $b=1, p=q=1$. See also Figure 3 with $b=1, p=2$ and $q=1$.

Special case: $|p+q|=1$. In this case, since the orthogonal projection to the $(u, v)$

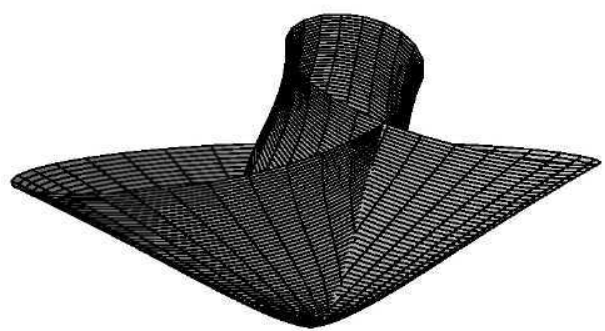

FIGURE 2 


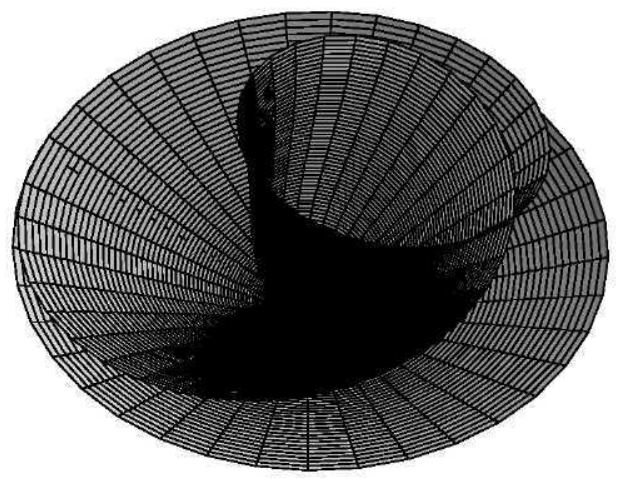

FIGURE 3.

plane restricted to each end is an injective map, we deduce that each end is embedded. We conclude that $\tilde{X}$ is a mean curvature one conformal immersion of $\boldsymbol{C}^{*}$ into $\boldsymbol{H}^{3}$ with two regular embedded ends and with finite total curvature. Observe that these surfaces are different from the immersed catenoids cousin, since they are locally associated to some singly periodic minimal surfaces of $\boldsymbol{R}^{3}$ and not to a catenoid (recall that $b \neq 0$ ). We can also infer from the expresion of $(\tilde{u}+i \tilde{v})(\zeta)$ that they are not rotational immersions. Finally, we infer from Theorem 2-3 of [SE-T, 1] that each end is asymptotic to a catenoid cousin, in both euclidean and hyperbolic meaning. See Figures 4, 5, 6, 7 and 8, where in each case we draw a half surface with $b=1$.

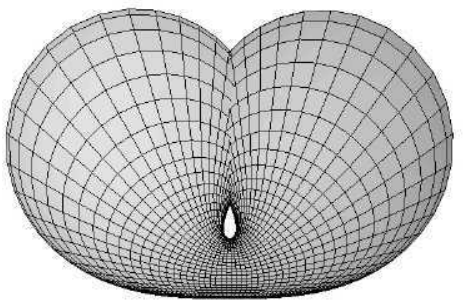

FIGURE 4. $p=-1, q=2$.

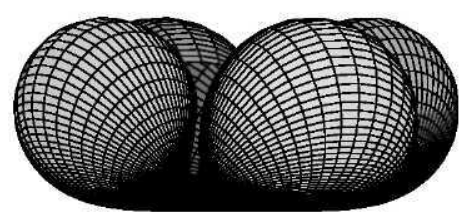

FIGURE $6 . \quad p=-3, q=4$.

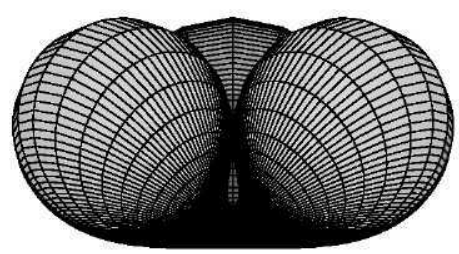

FIGURE 5. $p=-2, q=3$.

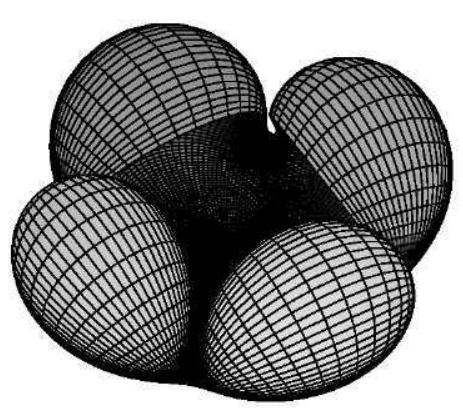

FIGURE 7. $p=-3, q=4$. 


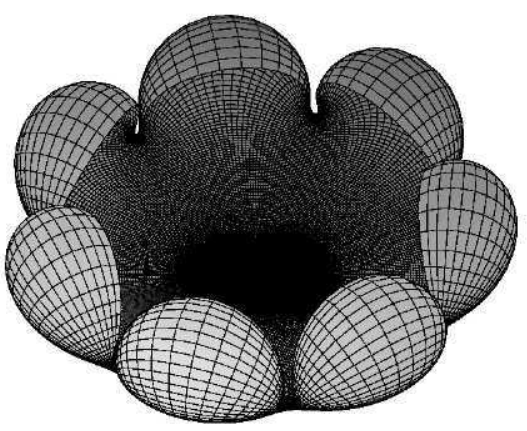

FIGURE $8 . \quad p=-6, q=7$.

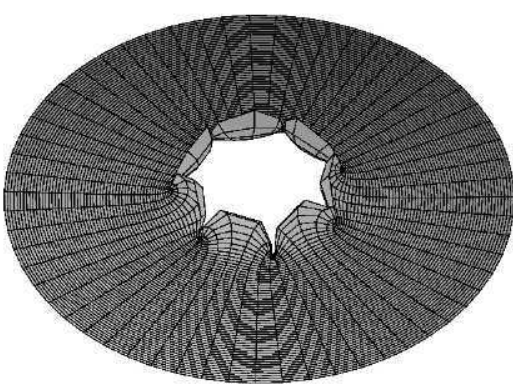

FIGURE 9. $p=-6, q=7$.

We draw in Figure 9 a piece of the other half part of the surface seen in Figure 8 .

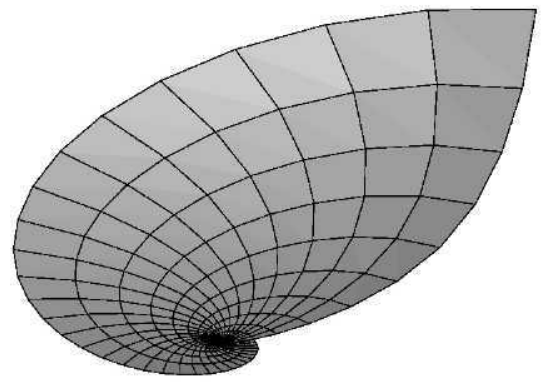

FIGURE 10

When $\gamma \in \boldsymbol{Q} \backslash\{0,-1,-1 / 2\}$, we observe that these surfaces were classified by Umehara and Yamada [U-Y-1, Theorem 6.2]. Their symmetry about a geodesic plane has been established in an alternative way by Rossman, Umehara and Yamada [R-U-Y, 3].

Fourth case: $\operatorname{Im}(\gamma) \neq 0$ and $\operatorname{Re}(\gamma) \neq 0$. In this case, $X(z+2 \pi i)=\left(H_{-4 \pi} \operatorname{Im}(\gamma) \circ\right.$ $\left.R_{4 \pi \operatorname{Re}(\gamma)}\right) X(z)$. That is, $X$ is invariant under a discrete subgroup of screw motions of $\boldsymbol{H}^{3}$ isometric to $\boldsymbol{Z}$. See Figure 10 , where $\gamma=-1+i$ and $b=1$.

REMARK 4-1. (1) Using the same arguments as in Example 2-12, we can state the following observation about the asymptotic boundary of $X(C)$ :

(i) When $\operatorname{Im}(\gamma) \neq 0$, then $\partial_{\infty} X\left(\boldsymbol{C}^{\prime}\right)=\partial_{\infty} \boldsymbol{H}^{3}$.

(ii) When $\gamma \in \boldsymbol{R} \backslash\{-1,0,-1 / 2\}$, then $\partial_{\infty} X(\boldsymbol{C})=\{0, \infty\}$.

(iii) When $\gamma=-1 / 2$, then $\partial_{\infty} X(\boldsymbol{C})=\{\infty\}$.

(2) Recently, Umehara and Yamada informed us that some of the above examples were known by them (personal communication). Nevertheless, the full geometric description we gave here is new. 
(3) It is worth mentioning that the authors have given in a previous work (see [SE$T, 2]$ ) families of examples of complete minimal surfaces in $\boldsymbol{H}^{3}$ invariant by a subgroup of discrete rigid motions (but not invariant by a one-parameter continuous family of such subgroups), namely a subgroup of either of the following isometries: hyperbolic, parabolic, elliptic. The techniques there are quite different, and are based on geometric analysis. That is, a combination of hyperbolic geometry with PDE methods, which enable us some flexibility.

\section{REFERENCES}

[B-SE, 1] J. L. BARBoSA AND R. SA EARP, Prescribed mean curvature hypersurfaces in $H^{n+1}(-1)$ with convex planar boundary, I, Geom. Dedicata 71 (1998), 61-74.

[B-SE, 2] J. L. BARbosa AND R. Sa EARP, Prescribed mean curvature hypersurfaces in $H^{n+1}$ with convex planar boundary, II, Séminaire de théorie spectrale et géometrie de Grenoble 16 (1998), 43 -79.

[B] R. BRYANT, Surfaces of mean curvature one in hyperbolic space, Asterisque 154-155 (1987), 321-347.

[C-H-R] P. COLLin, L. HaUswirTh AND H. RosenberG, The geometry of finite topology Bryant surfaces, Ann. of Math. (2) 153 (2001), 623-659.

[G-G] M. E. GALVÃo AND C. C. GóES, A Weierstrass type representation for surfaces in hyperbolic space with mean curvature one, Note Mat. 18 (1998), $43-61$.

[K] K. KENMOTSU, Weierstrass formula for surfaces of prescribed mean curvature, Math. Ann. 20 (1979), $177-183$.

[L] B. Lawson, Complete minimal surfaces in $S^{3}$, Ann. of Math. (2) 92 (1970), 335-374.

[L-Roit] L. L. DE LiMA AND P. RoITMAN, Constant mean curvature one surfaces in hyperbolic 3-space using the Bianchi-Calò method, An. Acad. Brasil. Ciênc. 74 (2002), 19-24.

[L-R] L. L. DE LIMA AND W. Rossman, On the index of mean curvature 1 surfaces in $H^{3}$, Indiana Univ. Math. J. 47 (1998), 685-723.

[N] B. NELLI, Hypersurfaces de courbure constante dans l'espace hyperbolique, Thèse de Doctorat Université Paris VII, 1995.

[Rev] Revue du Palais de la Découverte (1995), no. 45, 106.

[R-S] W. Rossman AND K. SATO, Constant mean curvature surfaces with two ends in hyperbolic 3-space, Experiment. Math. 7 (1998), 101-119.

[R-U-Y, 1] W. Rossman, M. UmeHARA AND K. YAmada, Irreducible constant mean curvature 1 surfaces in hyperbolic space with positive genus, Tôhoku Math. J. 49 (1997), $449-484$.

[R-U-Y, 2] W. ROSSMAN, M. UMEHARA AND K. YAMADA, Constructing mean curvature 1 surfaces in $\boldsymbol{H}^{3}$ with irregular ends, Preprint.

[R-U-Y, 3] W. Rossman, M. Umehara and K. Yamada, Mean curvature 1 surfaces in hyperbolic 3-space with low total curvature, Preprint; math.DG/0008015.

[S] A. J. SMALL, Surfaces of constant mean curvature 1 in $\boldsymbol{H}^{3}$ and algebraic curves on a quadric, Proc. Amer. Math. Soc. 122 (1994), 1211-1220.

[SE-T, 1] R. SA EARP AND E. TOUBIANA, On the geometry of constant mean curvature one surfaces in hyperbolic space, Illinois J. Math. 45 (2001), 371- 401.

[SE-T, 2] R. SA EARP AND E. TOUbIANA, Existence and uniqueness of minimal graphs in hyperbolic space, Asian J. Math. 4 (2000), 669-694.

[SE-T, 3] R. SA EARP AND E. TOUBIANA, Some applications of maximum principle to hypersurfaces theory in euclidean and hyperbolic space, New Approaches in Nonlinear Analysis (T. M. Rassias, Hadronic Press Inc, Florida, USA, 183 -202, eds.), 1999.

[SE-T, 4] R. S A EARP AND E. TOUBIANA, Introduction à la géométrie hyperbolique et aux surfaces de Riemann, Diderot Editeur 1997, Second edition, Cassini, Paris, to appear. 
[SE-T, 5] R. SA EARP AND E. ToubianA, Meromorphic data for mean curvature one surfaces in hyperbolic space II, Clay Math. Proc. 3 (2003), 521-540.

[SE-T, 6] R. SA EARP AND E. TOUBIANA, A Weirstrass-Kenmotsu formula for prescribed mean curvature surfaces in hyperbolic space, Séminaire de Théorie Spectrale et Géométrie de 1'Institut Fourier de Grenoble 19 (2001), 9-23.

[U-Y, 1] M. UMEHARA AND K. YAMADA, Complete surfaces of constant mean curvature-1 in the hyperbolic 3-space, Ann. of Math. 137 (1993), 611-638.

[U-Y, 2] M. UMEHARA AND K. YAMADA, A parametrization of the Weierstrass formulae and perturbation of some minimal surfaces in $R^{3}$ into the hyperbolic 3-space, J. Reine Angew. Math. 432 (1992), 93-116.

[U-Y, 3] M. UMEHARA AND K. YAMADA, Surfaces of constant mean curvature $c$ in $H^{3}\left(-c^{2}\right)$ with prescribed Gauss map, Math. Ann. 304 (1996), 203-224.

PONTIFÍCIA UNIVERSidAde CATÓLICA - RIO DE JANEIRO DEPARTAMENTO DE MATEMÁtica

RUA MARQUÊS DE SĀo VICENTE 225

22 453-900 RIO DE JANEIRO

BRAZIL

E-mail address: earp@mat.puc-rio.br
UNIVERSITÉ PARIS VII

DÉPARTEMENT DE MATHÉMATIQUES

2, PLACE JUSSIEU

75251 PARIS CEDEX 05

FRANCE

E-muil address: toubiana@math.jussieu.fr 